Ann. Génét. Sél. anim., I978, 10 (3), 403-44I.

\title{
Etude conjointe de l'intérêt de la sélection sur la prolificité et de l'utilisation d'une lignée mâle spécialisée chez le Porc
}

\author{
J.-M. ELSEN et P. SELLIER \\ Station de Génétique quantitative et appliquée, \\ Centre national de Recherches zootechniques, I.N.R.A. \\ 78350 Jouy-en-Josas
}

\begin{abstract}
Résumé
Nous avons étudié conjointement l'optimisation de certaines décisions concernant l'amélioration génétique d'une population porcine qui présente la structure pyramıdale de diffusion du progrès génétique (sélection, multiplication, production) et où le croisement terminal est un croisement à double étage avec des truies Large White $(\mathrm{LW}) \times$ Landrace Français $(\mathrm{LF})$. L'effectif de ces truies $F_{1}$ a été supposé constant et les effectifs de reproducteurs des étages supérieurs ont été ajustés aux besoins de renouvellement de l'étage de production, la monte naturelle étant seule utilisée. L'étude a porté sur deux types de choix :

- choix de la lignée paternelle du croisement terminal : nous avons comparé le croisement en retour (avec utilisation égale de verrats $\mathrm{LW}$ et $\mathrm{LF}$ ) et trois croisements triples avec une lignée "mâle "spécialisée : Landrace Belge, Piétrain et souche Large White "viande "distincte de la souche "femelle ";

- choix du plan de sélection des noyaux "femelles " (LW et $\left.L_{L} F\right)$ : plus précisément recherche de l'optimum pour les taux de sélection sur la prolificité (avec un indice de sélection de précision variable) et pour les taux de renouvellement des reproducteurs mâles et femelles.

Le critère à maximiser est le bénéfice actualisé retiré de la production de viande de porc dans l'ensemble de la population. Ce bénéfice, estimé pour diverses valeurs de la durée et du taux d'actualisation, a été décomposé en un "gain dû au croisement " et un "gain dû à la sélection "; dans ce dernier gain, on peut distinguer des recettes dues à la sélection sur la performance de production, des recettes dues à la sélection sur la prolificité et des recettes dues à l' effet conjoint de ces deux sélections.

Avec les hypothèses que nous avons retenues pour les paramètres fixés du modèle, les principales conclusions sont les suivantes :

- la meilleure politique de sélection dans les noyaux "femelles " dépend du type de croisement terminal. En particulier la sélection sur la prolificité ne se justifie pratiquement jamais dans le cas du croisement en retour, alors qu'elle se justifie dans le cas des croisements triples où l'application des taux optimaux de sélection sur la taille de portée permet d'accroître le gain dû à la sélection, par rapport à la situation où la prolificité est ignorée dans la sélection : cet accroissement peut aller jusqu'à 6 p. Ioo;

- le gain dû au croisement ne suffit pas à caractériser le mérite d'une stratégie de croisement. Ainsi le croisement triple avec le Landrace Belge est la meilleure stratégie de ce point de vue mais si l'on se réfère au bénéfice total calculé en supposant que, dans chaque cas, le plan optimal de sélection est appliqué, le croisement triple avec la souche Large White "viande " donne les meilleurs résultats, du fait d'une meilleure valorisation globale des actions de sélection, sur la période de 15 ans considérée.
\end{abstract}




\section{Introduction}

Le large développement de l'insémination artificielle, le petit nombre de centres de décision et l'importance des capitaux mis en jeu, ainsi que la longueur des intervalles de génération et donc du délai d'obtention des recettes dues à la sélection, sont probablement les causes essentielles de l'intérêt accordé depuis plusieurs années aux études économiques sur les schémas de sélection applicables à l'espèce bovine. A la suite de Poutous et VISSAC (I962), de nombreux auteurs ont tenté de "modéliser " ces schémas afin de déterminer les conditions de leur rentabilité maximale, en faisant généralement appel aux techniques d'actualisation : la sélection y est considérée comme une opération financière qui implique des coûts (investissement et fonctionnement) et permet la perception, à plus ou moins long terme, de recettes correspondant à l'accroissement de productivité chez les descendants des reproducteurs sélectionnés.

Dans les autres espèces, de telles études sont encore rares, bien que les principes de ces recherches et les méthodes utilisées pour les mener à bien leur soient aisément transposables. Dans le cas particulier du Porc, depuis les premiers travaux de Lush (1937) et de Dickerson et HAZEL (I944), de nombreuses études, dont les principales sont passées en revue par LEGAULT et OLLIVIER (I974), ont été consacrées à la recherche des meilleurs plans de sélection sans étudier de façon globale leur rentabilité mais en considérant seulement des aspects purement génétiques, l'objectif recherché étant le plus souvent l'obtention du progrès génétique annuel maximum dans les élevages de sélection. Il s'agit alors de trouver la combinaison optimum de trois facteurs qui sont la précision de l'estimation de la valeur génétique des candidats à la sélection, l'intensité de sélection et l'intervalle de génération, en faisant implicitement l'hypothèse qu'aucune limite n'est imposée au recueil de l'information nécessaire à la sélection. Dans l'approche "généticoéconomique " qui sera utilisée ici, il est tenu compte en plus, d'une part du co ît de la création du progrès génétique, d'autre part du mode de diffusion de ce progrès à l'ensemble de la population soumise à sélection. Certaines études réalisées dans le passé sur l'optimisation de la sélection se rattachent, au moins de façon partielle, à ce type de démarche. Ainsi Smith (I959), recherchant la meilleure utilisation d'une capacité de contrôle donnée ( $=$ coût) dans les stations de sélection, a notamment montré qu'il vaut mieux restreindre les opérations de sélection à un nombre limité d'élevages (système du noyau), plutôt que de les "diluer " dans l'ensemble des élevages de production. Le mode de diffusion de l'amélioration du noyau de sélection jusqu'au stade des élevages commerciaux a été décrit dans diverses situations par BICHARD (I97I) et certains aspects de cette diffusion ont été développés par Brchard et WII,SON (I974). Dans le cas du contrôle en ferme, LEGAULT (I970a) a analysé l'intérêt d'un choix précoce sur la prolificité de la mère avant la sélection individuelle sur la vitesse de croissance et l'épaisseur du lard dorsal, en tenant compte du coût respectif de ces deux sélections pour l'éleveur. Par ailleurs, l'approche économique a déjà été utilisée dans les recherches sur l'optimisation des plans de croisement chez le Porc, d'abord en se limitant au seul étage terminal du plan de croissement (MoAv, I966), puis en considérant l'ensemble de la population soumise au croisement (JAKUBEC et FEwSON, 1970; DicKERSON, I973; BRUN, I974; BICHARD, I977).

Dans la présente étude, nous avons tenté d'appliquer ce type d'approche génético-économique à l'étude des choix à faire à la fois en matière de plan de croisement et en matière de plan de sélection et à l'étude de l'" interaction " pos- 
sible entre ces deux types de choix, dans le cas d'une population porcine présentant la structure pyramidale classique de diffusion du progrès génétique. Nous avons supposé, comme dans toutes les études de ce genre, que les intérêts des agents économiques impliqués dans la réalisation du schéma d'amélioration génétique étaient convergents et qu'on pouvait donc raisonner comme si toutes les décisions étaient prises par un maître-d'œuvre unique dont l'objectif est d'optimiser, par des moyens génétiques, la rentabilité de la production de viande de porc dans l'ensemble de la population considérée.

\section{I. - Position du problème et définition des objectifs de l'étude}

Comme l'indique Cunningham (I973), il faut pour établir un schéma d'amélioration génétique : (I) déterminer les caractères d'intérêt économique et leurs mesures possibles, (2) connaître les paramètres génétiques correspondants (héritabilités, corrélations génétiques, effets d'hétérosis), (3) choisir les races intervenant dans le plan de croisement éventuel. Le schéma sera alors défini par le plan de croisement pratiqué (éventuellement la race pure) et par le plan de sélection mis en œuvre dans chaque race (ou souche) intervenant dans le croisement.

Chez le Porc, les performances à améliorer en priorité sont :

- d'une part la prolificité, composante essentielle de la productivité numérique des truies (nombre de produits/truie/an); la taille de portée est un caractère peu héritable (LEGaulT, I970 $b$; StRANG et KING, 1970) mais soumis à des effets d'hétérosis individuel et maternel importants (SELLIER, I976b);

- d'autre part, l'efficacité alimentaire de l'animal en croissance pour la production de viande maigre (performance dite de production); la variance génétique des caractères de croissance et de composition corporelle est essentiellement additive, comme 1'a montré par exemple OLLIVIER (r970).

Par ailleurs, l'absence de liaison génétique entre la taille de portée et la performance de production est l'hypothèse de travail la plus vraisemblable, ainsi que le montrent les estimations de corrélations génétiques et les résultats de plusieurs expériences de sélection (GRAY et al., I965; HETZER et MILLER, I970; LEGAULT, I97I $b$; MORRIS, I975; WeBb et KING, I976; GARNETT et RAHNEFELD, I976; NEWTON et al., 1977; ENGLAND et al., 1977) et bien que d'autres expériences de sélection semblent indiquer le contraire (BERRUECOS et al., I970; VANGEN, I972, I977).

A l'heure actuelle, dans la définition des schémas d'amélioration génétique du Porc, deux orientations se dessinent nettement :

I. - Utilisation, comme lignée maternelle du croisement terminal, de truies issues d'un croisement à 2 voies $\left(F_{1}\right)$ qui est presque toujours de type Landrace $\times$ Large White (Yorkshire) dans les pays européens (SELLIER, I974).

2. - Utilisation du contrôle individuel pour la sélection de la lignée paternelle sur les seuls caractères de croissance et de composition corporelle (LEGAULT et OliLivier, I974).

La validité de ces deux choix repose sur des bases théoriques et expérimentales solides et il y a peu de chances qu'ils soient remis en cause dans un proche avenir : nous les retiendrons donc dans cette étude. 
Par contre des incertitudes subsistent sur deux points :

I. - Le plan de sélection à appliquer dans les lignées "femelles " utilisées pour la production des truies $F_{1}$ : faut-il introduire la prolificité dans les critères de sélection de ces lignées?

2. - Le type génétique à utiliser comme lignée paternelle : faut-il faire appel à une lignée "mâle" spécialisée?

Nous avons cherché à donner des éléments de réponse à ces deux grandes questions en considérant une population de structure pyramidale comprenant des noyaux de races pures où est créé le progrès génétique (étage de sélection), des troupeaux où sont produites les truies $F_{1}$ (étage de multiplication) et des troupeaux commerciaux où naissent et sont engraissés les produits terminaux (étage de production). Le nombre de femelles $F_{1}$ en reproduction dans les élevages commerciaux a été fixé à roo o0o. Nous avons pris comme effectifs de reproducteurs dans les étages de multiplication et de sélection les effectifs minimaux nécessaires au renouvellement de ces roo ooo femelles et des mâles qui leur sont accouplés, en admettant que l'insémination artificielle n'est pas utilisée. Tous ces effectifs sont supposés constants dans le temps.

(a)

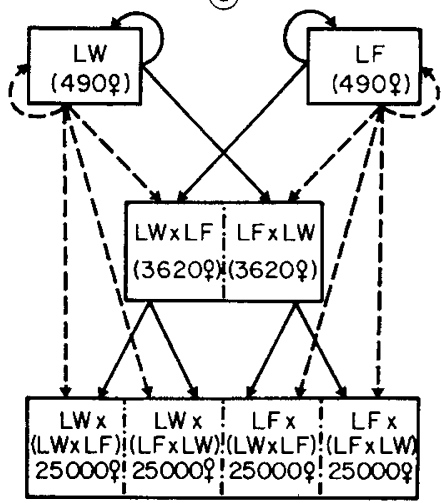

(b)

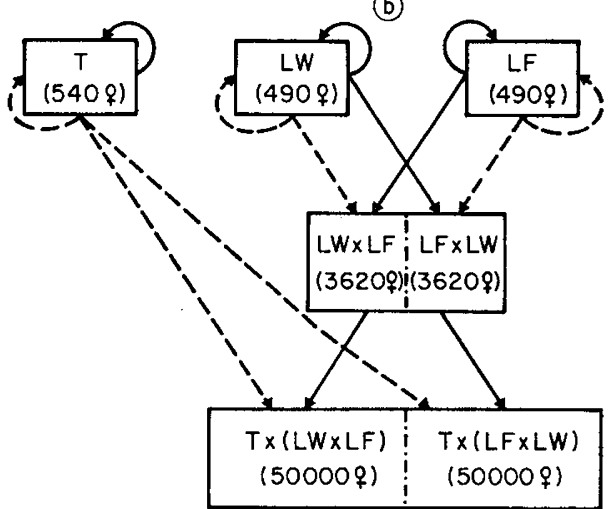

FIG. I. - Structure de la population et flux de veproducteurs dans le cas du croisement en vetour (a) et dans le cas d'un croisement triple (b)

Structure of the population and transfers of breeding animals in backcrossing (a) and in a three-way crossing (b)

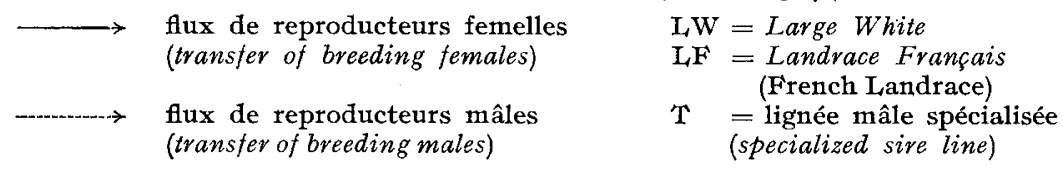

De façon plus précise, les objectifs de notre étude se rattachent à trois types de problèmes :

\section{I. - Choix de la lignée paternelle du croisement terminal}

De ce point de vue, deux grandes options peuvent être envisagées : le croisement en retour et le croisement triple. Dans le croisement en retour, les femelles $F_{1}$ sont accouplées à des mâles Large White et Landrace Français issus des mêmes souches que leurs propres parents (fig. I $a$ ) : cette solution est dans la pratique la 
plus souvent retenue à l'heure actuelle et nous avons supposé que la structure démographique de la population à l'instant initial correspondait à ce plan de croisement. Dans le croisement triple, on utilise une lignée mâle spécialisée pour la production de viande : les trois variantes envisagées ici sont le Piétrain, le Landrace Belge et une souche Large White "viande " distincte de la souche Large White "femelle" (fig. I $b$ ).

\section{2. - Prise en compte de la prolificité dans la sélection des souches "femelles"}

Bien que d'un point de vue strictement génétique, il soit toujours préférable d'utiliser un indice de sélection pour l'amélioration simultanée de plusieurs caractères (HAZEL et LUSH, I942), nous avons envisagé une sélection en deux étapes : d'abord (au sevrage) sur la prolificité (choix sur ascendance), ensuite (à Ioo kg) sur la performance de production (choix sur un indice de contrôle individuel combinant vitesse de croissance et épaisseur de lard dorsal). Cette méthode a l'avantage d'être moins coûteuse puisque les animaux éliminés lors du premier choix ne sont pas contrôlés individuellement sur leur performance de production, le coût de ce dernier contrôle étant lié surtout à la non-castration des mâles. Notons que même avec cette méthode, il aurait été préférable, d'un point de vue génétique, d'utiliser, pour le second stade de sélection, un indice global regroupant performance de production et prolificité mais que, pour des raisons de volume des calculs, nous avons dû abandonner cette possibilité. Le problème consiste alors à rechercher les meilleurs taux de sélection sur la prolificité à appliquer dans les souches "femelles " Large White et Landrace Français, ces taux pouvant varier avec le sexe des candidats. Nous avons également étudié l'intérêt d'une précision accrue de l'estimation de la valeur génétique pour la prolificité en tenant compte non seulement de l'information relative à la mère du candidat à la sélection mais aussi de l'information relative à la mère du candidat à la sélection mais aussi de l'information relative à un certain nombre d'autres apparentées contemporaines de la mère.

\section{3. - Choix des taux de renouvellement dans les noyaux "femelles" de sélection}

Nous avons tenté de déterminer de façon approchée les taux de renouvellement optimaux des reproducteurs de ces noyaux en comparant quatre situations définies par une ou deux périodes de saillie pour les mâles, avec, dans chaque cas, une ou deux mises bas par femelle. Les solutions à taux de renouvellement le plus grand ont 1'avantage de conduire à un intervalle de génération plus court alors que les solutions à taux de renouvellement plus faible permettent des pressions de sélection plus élevées et, dans le cas de deux mises bas par femelle, une plus grande précision du choix sur la prolificité. Ajoutons que dans le cas du noyau de sélection de la lignée mâle, une seule situation a été envisagée, à savoir une période de saillie pour les mâles et une mise bas pour les femelles : elle correspond approximativement à l'optimum trouvé par OLIIVIER (I974) pour la sélection individuelle sur les caractères de croissance et de carcasse.

En pratique, nous sommes partis d'une situation définie de la façon suivante : la population considérée présente la structure démographique du croisement en retour à l'équilibre (fig. I $a$ ) et il est supposé qu'elle n'a été soumise antérieurement à aucune sélection. A l'année $\mathrm{o}$, les opérations de sélection commencent et, en ce qui concerne le plan de croisement, soit on continue à pratiquer le croisement en retour, soit on lui substitue progressivement l'un des croisements triples : 
le noyau de sélection de la lignée mâle spécialisée est créé et à partir de ce moment, le renouvellement des mâles des troupeaux de production se fait avec des mâles issus de ce noyau : dans le cas du croisement triple, il y a donc une phase de transition avant d'obtenir le nouvel équilibre démographique.

Le problème, tel que nous l'avons posé, a été décomposé en deux étapes distinctes.

\section{TABLEAU I}

Définition des variantes des facteurs étudiés

Definition of the variants of the factors investigated

\begin{tabular}{|c|c|c|}
\hline $\begin{array}{c}\text { Facteur } \\
\text { (factor) }\end{array}$ & de $\begin{array}{c}\text { Nombre } \\
\text { variantes } \\
\text { (no. of } \\
\text { variants })\end{array}$ & $\begin{array}{l}\text { Définition des variantes } \\
\text { (definition of the variants) }\end{array}$ \\
\hline $\begin{array}{l}\text { I - plan de croisement: } \\
\text { type génétique du } \\
\text { verrat pour le croi- } \\
\text { sement terminal } \\
\text { (crossing plan: breed } \\
\text { of boar in the termi- } \\
\text { nal cross) }\end{array}$ & 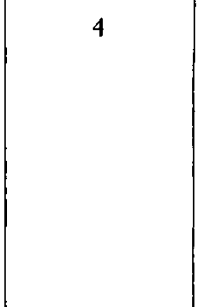 & $\begin{array}{l}\text { I } a \text { croisements en retour avec Large White et Landrace } \\
\text { Français. } \\
\text { (backcrosses to Large White and French Landrace) } \\
\text { Ib croisement triple avec Pietrain (3-way cross with } \\
\text { Pietrain) } \\
\text { Ic croisement triple avec Landrace Belge (3-way cross } \\
\text { with Belgian Landrace) } \\
\text { Id croisement triple avec souche Large White "viande } \\
\text { (3-way cross with Large White sire strain) }\end{array}$ \\
\hline $\begin{array}{l}\text { II - Source d'informa- } \\
\text { tion pour la sélec- } \\
\text { tion sur la prolifi- } \\
\text { cité dans les no- } \\
\text { yaux " femelles" } \\
\text { (information used } \\
\text { for selection on lit- } \\
\text { ter size in the "fe- } \\
\text { male» nucleus herds) }\end{array}$ & 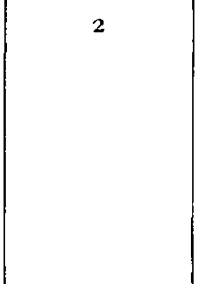 & $\begin{array}{l}\text { II } a \text { mère du candidat } \\
\quad \text { (dam of the candidate }) \\
\text { II } b \text { mère }+ \text { tantes et } \mathrm{I} / 2 \text { tantes du candidat } \\
\quad(\text { dam }+ \text { aunts and half-aunts of the candidate })\end{array}$ \\
\hline $\begin{array}{l}\text { III-Situations de re- } \\
\text { nouvellement dans } \\
\text { les noyaux de sé- } \\
\text { lection "femelles" } \\
\text { (rules for replace- } \\
\text { ment in the "fema- } \\
\text { le " nucleus herds) }\end{array}$ & 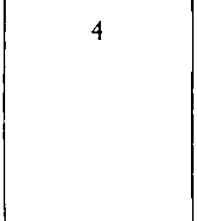 & 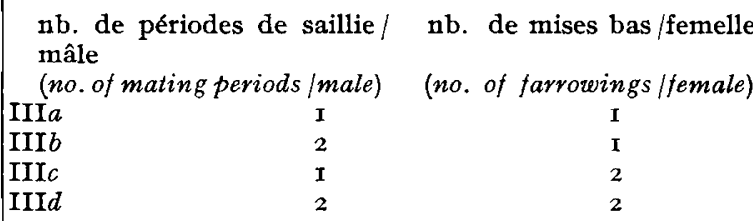 \\
\hline $\begin{array}{l}\text { IV-Taux d'actualisa- } \\
\text { tion annuel } \\
\text { (annual discounting } \\
\text { rate) }\end{array}$ & 3 & $\begin{array}{llll}\text { IV } a & \text { o } & \text { p. } & \text { 100 } \\
\text { IV } b & \text { Io } & \text { p. } & \text { 100 } \\
\text { IV } c & 20 & \text { p. } & \text { 100 }\end{array}$ \\
\hline $\begin{array}{l}\text { V-Durée d'actualisa- } \\
\text { tion en semestres } \\
\text { (length of discount- } \\
\text { ing period in half- } \\
\text { years) }\end{array}$ & 3 & $\begin{array}{ll}\mathrm{V} a & \text { 10 } \\
\mathrm{V} b & 20 \\
\mathrm{~V} c & 30\end{array}$ \\
\hline
\end{tabular}


La première étape consiste à déterminer l'expression algébrique du critère de comparaison utilisé (le bénéfice actualisé défini ci-dessous) pour les différentes situations envisagées. Les variantes étudiées pour chacun des 5 facteurs pris en considération sont données dans le tableau I. Au total il y a 288 situations possibles dans ce "dispositif factoriel ". En réalité toutes ces situations n'ont pas été envisagées et l'étude a été menée de la façon suivante. Dans un premier temps, l'influence des facteurs II, III, IV et $\mathrm{V}$ a été étudiée de façon complète pour les seules variantes I $a$ (croisement en retour) et $\mathrm{I} b$ (croisement triple avec le Piétrain) du plan de croisement. L'étude des variantes Ic et Id (croisement triple avec le Landrace Belge et la souche Large White "viande ") a été abordée ensuite en considérant seulement, pour les autres facteurs, les variantes qui semblaient $a$ priori les plus intéressantes au vu des résultcts obtenus précédemment.

La seconde étape consiste à rechercher, pour chaque situation, les valeurs des taux de sélection sur la prolificité maximisant le critère de comparaison, compte tenu de contraintes sur ces taux. Cette étape est donc un problème classique d'optimisation sous contrainte, le nombre de variables et de contraintes s'élevant dans notre cas à 6 et $\mathrm{I} 4$ respectivement.

\section{II. - Méthodes et hypothèses}

\section{A. - Critère de comparaison}

Le critère de comparaison entre schémas est le bénéfice actualisé, estimé pour diverses valeurs de la durée et du taux d'actualisation. Dans sa forme la plus générale, ce bénéfice s'écrit :

$$
\mathrm{B}=\mathrm{R}-\mathrm{C}=\sum_{t=1}^{T} \theta^{t}\left(\mathrm{R}_{t}-\mathrm{C}_{t}\right)
$$

où $T$ et $\theta$ sont la durée et le facteur d'actualisation, $\mathrm{R}_{t}$ et $\mathrm{C}_{t}$ sont respectivement les recettes et les coûts intervenant à l'instant $t$.

Nous avons adopté comme unité de temps le semestre. Cette unité est bien adaptée à l'espèce porcine puisqu'elle correspond approximativement à la fois à l'intervalle entre mises bas et à l'âge d'abattage d'un porc de $100 \mathrm{~kg}$. Le facteur d'actualisation $\theta$ est donc lié au taux annuel d'actualisation $a$ par la relation $\theta=(\mathbf{I}+a)^{-1 / 2}$.

\section{B. - Estimation des recettes}

\section{r. Généralités}

Nous désignons ici par " recettes" la marge brute totale retirée de la vente des animaux pour la boucherie. Ces animaux se répartissent en cinq catégories :

- les reproducteurs réformés de tous les étages; les recettes provenant de leur vente ont été traitées comme des " coûts négatifs" (voir plus loin);

- les produits terminaux de l'étage de production (femelles et mâles castrés de roo $\mathrm{kg}$ );

- les produits mâles $F_{1}$ nés dans les élevages de multiplication et vendus comme mâles castrés de roo $\mathrm{kg}$; 
- les candidats à la sélection non retenus pour le renouvellement dans les noyaux de sélection : ce sont des femelles et des mâles entiers, ainsi que, pour une partie, des mâles castrés dans les noyaux "femelles " (il est supposé que les mâles éliminés lors du choix fait au sevrage sur l'indice de prolificité sont vendus comme mâles castrés à $100 \mathrm{~kg}$ );

- le surplus de femelles $F_{1}$ par rapport aux besoins de renouvellement des élevages de production : ce surplus, dont l'existence est liée à l'hypothèse de constance de l'effectif de reproductrices $F_{1}$ dans l'étage de production, est nul à l'instant initial et va augmenter avec le temps dans l'éventualité d'une sélection sur la prolificité.

La marge brute totale est donc fonction de l'effectif de porcs vendus, qui varie avec la prolificité, et de la marge unitaire par porc vendu. Par marge unitaire, quantité dont le mode de calcul sera précisé plus loin, nous entendons le bénéfice retiré de l'engraissement d'un porc sans tenir compte de ce qui est imputable à la mère dans le prix de revient du porcelet de $25 \mathrm{~kg}$. Dans le cas des femelles et des mâles castrés, la marge unitaire dépend à la fois du type génétique du porc et de 1'année de naissance de ses parents : à un instant donné et dans un étage donné, les descendants des truies ou verrats les plus jeunes sont génétiquement supérieurs de ce point de vue, du fait de la sélection. Dans le cas des jeunes mâles entiers vendus à l'issue du contrôle individuel, la marge unitaire est plus faible dans les conditions actuelles du marché et nous l'avons supposée indépendante du type génétique et non modifiée par la sélection. La taille de portée d'une truie varie, pour les mêmes raisons, avec son type génétique, l'année de naissance de ses parents et aussi avec son âge, dans la mesure où celui-ci a un effet sur la prolificité.

A l'instant $t$, la population peut être décomposée en cohortes d'animaux homogènes vis-à-vis de leur étage d'origine, de leur type génétique, de leur sexe, de leur âge, de 1'âge de leur mère et de leur "destinée " (reproducteur ou porc abattu). Parmi ces cohortes, on distinguera en particulier deux sous-ensembles : le sousensemble $I$ constitué par les cohortes de produits abattus à roo $\mathrm{kg}$ et le sous-ensemble $J$ constitué par les cobortes de femelles en reproduction. A chaque cohorte de produits indicée $i(i \in I)$ correspond à la date $t$ une valeur moyenne de la marge unitaire $y_{(i) t}$ et une valeur moyenne de la prolificité de leurs mères $x_{(j) t}(j \in J)$, estimée par la taille de portée au sevrage. Dès lors les recettes perçues à la date $t$ s'écrivent :

$$
\mathrm{R}_{t}=\sum_{i \in I} y_{(i) t}\left[\sum_{j \in J}\left(k \mathrm{~N}_{i j} x_{(j) t}-\mathrm{A}_{i j}\right)\right]
$$

où

$k$ est le taux de survie entre le sevrage et roo $\mathrm{kg}$;

$\mathrm{N}_{i j}$ est le nombre de mères de la cohorte $j$ qui donnent naissance à des porcs de la cohorte $i$;

$\mathrm{A}_{i j}$ est le nombre d'individus appartenant à la cohorte $i$, issus des mères de la cohorte $j$ et retenus pour le renouvellement $\left(A_{i j}\right.$ peut être nul).

En notant $\mathrm{R}_{1 t}=\sum_{i \in I} y_{(i) t}\left(\sum_{j \in J} \mathrm{~N}_{i j} y_{(j) t}\right)$ et $\mathrm{R}_{2 t}=\sum_{i \in I} y_{(i) t}\left(\sum_{j \in J} \mathrm{~A}_{i j}\right)$, les recettes totales sont :

$$
\mathrm{R}=\sum_{t=1}^{T}\left(\mathrm{R}_{1} t-\mathrm{R}_{2} t\right) \theta^{t}
$$




\section{2. - Estimation des marges unitaires et de la prolificité des truies}

\section{a) Méthode d'estimation:}

La marge unitaire et la prolificité évoluent dans le temps, du fait de la sélection : elles ont été estimées à 1'aide du modèle développé par HiLL (I974) et EiLSEN et Mocouot (I974). Ce modèle décrit l'évolution, période par période, du niveau génétique d'une population soumise à sélection et dont les générations se chevauchent, à l'aide de l'algorithme suivant : $\mathrm{G}_{t}$ étant un vecteur dont chaque élément $\mathrm{G}_{t(i)}$ est la valeur génétique additive moyenne des animaux de la cohorte $i$ à la période $t, \Delta \mathrm{G}_{t}$ étant un vecteur dont chaque élément $\Delta \mathrm{G}_{t(i)}$ est la supériorité des animaux sélectionnés dans la cohorte $i$ par rapport aux candidats à la sélection de cette même cohorte et $\mathrm{D}_{t}$ étant une matrice de transition qui permet de passer de la période $t$ à la période $t+I$ et dépend des pyramides des âges des reproducteurs, le vecteur $\mathrm{G}_{t+{ }_{I}}$ est déduit du vecteur $\mathrm{G}_{t}$ par l'opération :

(I) $\mathrm{G}_{t+\mathrm{I}}=\mathrm{D}_{t} \mathrm{G}_{t}+\Delta \mathrm{G}_{t}$

Le modèle étant linéaire, seuls les effets additifs sont pris en compte. Le vecteur $\mathrm{P}_{t+I}$ des valeurs phénotypiques $\mathrm{P}_{t+I(i)}$ des animaux de la cohorte $i$ au temps $t+I$ est donné par l'équation :

(2) $\mathrm{P}_{t+I}=\mathrm{G}_{t+I}+\mathrm{E}=\mathrm{D}_{t} \mathrm{G}_{t}+\Delta \mathrm{G}_{t}+\mathrm{E}$

où $\mathrm{E}$ est le vecteur des écarts à l'additivité que nous avons supposés invariants dans le temps. Ces écarts comprennent d'une part les effets d'hétérosis sur les performances de production et de reproduction, d'autre par les effets génétiques maternels et paternels et l'effet de 1'âge de la truie sur la taille de portée, traitée ici comme un caractère de la mère et non du produit. Appliquée à la marge unitaire $(y)$ et à la prolificité $(x)$, l'équation (2) devient :

(3) $y_{t+\boldsymbol{x}}=\mathrm{G}_{\mathrm{y}, t+\boldsymbol{I}}+\mathrm{E}_{\mathrm{y}}$ avec $\mathrm{G}_{\mathrm{y}, t+\boldsymbol{I}}=\mathrm{D}_{t} \mathrm{G}_{\mathrm{y}, t}+\Delta \mathrm{G}_{\mathrm{y}, t}$

(4) $x_{t^{+} I}=\mathrm{G}_{\mathbf{X}, t+t}+\mathrm{E}_{\mathbf{X}}$ avec $\mathrm{G}_{\mathbf{X}, t+I}=\mathrm{D}_{t} \mathrm{G}_{\mathbf{X}, t}+\Delta \mathrm{G}_{\mathbf{X}, t}$

Nous avons supposé par ailleurs que le schéma de sélection se répète identique à lui-même dans le temps, donc que $\Delta \mathrm{G}_{\mathbf{y}}, t, \Delta \mathrm{G}_{\mathbf{x}}, t$ et $\mathrm{D}_{t}$ sont des constantes notées $\Delta \mathrm{G}_{\mathbf{y}}, \Delta \mathrm{G}_{\mathbf{x}}$ et $\mathrm{D}$. Cette hypothèse implique en particulier que les pressions de sélection ne varient pas (leur accroissement possible dû à la réponse à la sélection sur la prolificité est supposé négligeable), que les paramètres génétiques des caractères sélectionnés restent les mêmes et que les pyramides des âges ne sont pas modifiées par la sélection.

Avec cette hypothèse, les relations (3) et (4) s'écrivent :

(5) $y_{t+\boldsymbol{x}}=\mathrm{D}^{t+x} \mathrm{G}_{\mathrm{y}, o}+\mathrm{D}_{t} \Delta \mathrm{G}_{\mathrm{y}}+\mathrm{E}_{\mathrm{y}}$

(6) $x_{t+\boldsymbol{x}}=\mathrm{D}^{t+x} \mathrm{G}_{\mathbf{X}, o}+\mathcal{D}_{t} \Delta \mathrm{G}_{\mathbf{X}}+\mathrm{E}_{\mathbf{X}}$

où $\mathfrak{D}_{t}=\mathrm{D}^{t}+\mathrm{D}^{t-I}+\ldots+\mathrm{I}$ (I est la matrice unité de même dimension que $\mathrm{D}$ ).

Ces valeurs comprennent donc une part due à la sélection $-\mathscr{D}_{t} \Delta \mathrm{G}_{\mathbf{y}}$ et $D_{t} \Delta \mathrm{G}_{\mathrm{x}}$ - et une part due au croisement $-\mathrm{D}^{t+I} \mathrm{G}_{\mathrm{y}, o}+\mathrm{E}_{\mathrm{y}}$ et $\mathrm{D}^{t+r} \mathrm{G}_{\mathrm{x}, o}+\mathrm{E}_{\mathrm{x}}$ Dans le cas du croisement en retour, qui est supposé en phase d'équilibre à l'instant initial, c'est-à-dire appliqué sous la même forme depuis un temps suffisant pour qu'à cet instant les effectifs des différents types d'animaux soient stables et que la valeur moyenne d'une cohorte ne varie plus, on a :

$$
\mathrm{G}_{\mathbf{y}, o}=\mathrm{DG}_{\mathbf{y}, o} \text { et } \mathrm{G}_{\mathbf{x}, o}=\mathrm{DG}_{\mathbf{x}, o}
$$


et les relations (5) et (6) deviennent :

(7) $y_{t+I}=y_{0}+D_{t} \Delta \mathrm{G}_{\mathrm{y}}$

(8) $x_{t+x}=x_{o}+\mathfrak{D}_{t} \Delta \mathrm{G}_{\mathbf{x}}$

Les valeurs $y_{t+I}$ et $x_{t++_{I}}$ sont donc fonction des niveaux initiaux $y_{0}$ et $x_{0}$ et des supériorités dues à la sélection $\Delta \mathrm{G}_{\mathbf{y}}$ et $\Delta \mathrm{G}_{\mathbf{x}}$.

Dans le cas du croisement triple, les relations (7) et (8) ne sont vraies en toute rigueur qu'à partir du quatrième semestre, époque à laquelle tous les verrats en service dans l'étage de production proviennent du noyau "mâle ». On a donc remplacé $x_{0}$ et $y_{0}$ par $x_{4}$ et $y_{4}$, et les valeurs correspondant aux trois premiers semestres ont été calculées pas à pas.

\section{TABLEAU 2}

Valeur initiale de la taille de portée au sevrage pour les différents types génétiques et effet du numéro de mise bas de la mère

Initial value of litter size at weaning in the different breeding groups and effect of dam parity

effet du type génétique $\dagger$

(effect of breeding group)

\begin{tabular}{|c|c|c|c|c|c|c|c|c|}
\hline \multirow[b]{2}{*}{$\begin{array}{l}\text { Type génétique de la portée } \\
\text { (breeding group of the litter) }\end{array}$} & \multicolumn{4}{|c|}{$\begin{array}{c}\text { races pures } \\
\text { (purebreds) }\end{array}$} & \multicolumn{4}{|c|}{$\begin{array}{l}\text { croisements } \\
\text { (crossbreds) }\end{array}$} \\
\hline & LW & $\mathrm{LF}$ & $\mathrm{LB}_{\mathrm{B}}$ & $\mathrm{P}$ & $\begin{array}{l}\mathrm{LW} \\
\times \mathrm{LF}\end{array}$ & $\begin{array}{l}\mathrm{LF} \\
\times \mathrm{LW}\end{array}$ & $\underset{\mathrm{F}_{1}}{\mathrm{LW}} \times$ & $\begin{array}{c}\mathrm{LF}, \mathrm{LB}, \mathrm{P} \\
\times \mathrm{F}_{1}\end{array}$ \\
\hline $\begin{array}{l}\text { Valeur moyenne pour la } \mathrm{r} \text { re } \\
\text { mise bas } \cdot \text { for the first } \\
\text { (mean value for } \\
\text { parity) }\end{array}$ & 8,15 & $8, \mathrm{I} 5$ & 7,20 & 7,20 & 8,55 & 8,55 & 9,35 & 8,75 \\
\hline
\end{tabular}

effet du numéro de mise bas de la mère

(effect of dam parity)

\begin{tabular}{|c|c|c|c|c|c|c|}
\hline $\begin{array}{l}\text { Numéro de mise bas } \\
\text { (parity) }\end{array}$ & $x$ & 2 & 3 & 4 & 5 & 6 \\
\hline $\begin{array}{l}\text { Ecart par rapport à la } \mathbf{I r} \\
\text { mise bas } \\
\text { (deviation from the first pa- } \\
\text { vity) }\end{array}$ & o & $+0,83 \S$ & $+\mathrm{I}, \mathrm{I} 5$ & $+\mathrm{I}, \mathrm{I} 5$ & $+\mathrm{I}, \mathbf{I} 3$ & $+0,97$ \\
\hline
\end{tabular}

$\dagger \mathrm{LW}=$ Large White, $\mathrm{L}_{\mathbf{F}}=$ Landrace Français, $\mathrm{LB}=$ Landrace Belge, $\mathbf{P}=$ Piétrain, $\mathbf{F}_{1}=\mathrm{LW}_{\mathrm{W}} \times$ $I_{\text {F }}$ et $\mathrm{L}_{1} \mathrm{~F} \times \mathrm{LW}$; pour les croisements, la race du père est donnée en premier (for crossbreds, the breed of sire is first given).

$\S+0,57$ pour le Landrace Belge et le Piétrain. 
b) Valeurs initiales des prolificités et des marges unitaires:

\section{a. Prolificités}

Les prolificités initiales ont été décomposées pour les besoins du modèle en $x_{o}=\mathrm{G}_{\mathrm{x}, o}+\mathrm{E}_{\mathrm{x}}$. Ces composantes ont été estimées à partir des données de Smith et KING (I964), VAN DE PAs et al. (I973) et LEGAULT et al. (I975) pour chaque type génétique et pour chaque âge de la truie. Les valeurs de la taille de portée au sevrage sont données au tableau 2 pour chaque type génétique, ainsi que l'effet du numéro de mise bas de la mère. On notera que la taille de portée est identique pour les femelles de race pure Large White et Landrace Français, comme pour les deux types de truies $F_{1}$, et qu'il existe un effet important de la race du père sur la taille des portées des truies $\mathrm{F}_{1}$, à l'avantage du Large White.

\section{ß. Marges unitaires}

Nous avons défini la marge du porc de roo $\mathrm{kg}$ comme la différence entre son prix de vente et son prix de revient propre, calculé sans tenir compte des frais d'entretien de la mère. Il est en effet apparu plus simple de dissocier pour le calcul du prix de revient d'un porc de Ioo $\mathrm{kg}$ le coût imputable au porc lui-même et la fraction du coût d'entretien de la mère qui lui serait imputée dans la méthode classique de calcul du prix de revient du porcelet (I,EGAULT, I97I $a$ ), fraction qui diminue dans le temps du fait de l'accroissement de la taille de portée; avec cette démarche, la marge unitaire est donc indépendante de la prolificité. Par exemple, la marge par porc de race Large White a été estimée à I30 F, en supposant un prix de vente de $435 \mathrm{~F}$, un prix de revient entre la naissance et le poids de $25 \mathrm{~kg}$ de $50 \mathrm{~F}$ et un coût de l'engraissement entre 25 et Ioo $\mathrm{kg}$ égal à $255 \mathrm{~F}$ (indice de consommation : $3,4 \mathrm{~kg}$ d'aliment $/ \mathrm{kg}$ de gain; prix de l'aliment : $0,8 \mathrm{~F} / \mathrm{kg}$; coûts autres qu'alimentaires supposés fixes et égaux à $5 \mathrm{I} F$ ). Les marges par porc ont été estimées pour les autres types génétiques, à partir des pondérations économiques données par MolENAT et al. (I974), soit en utilisant directement des résultats de comparaisons entre plans de croisement (SELI,IER, I975, I976a), soit en partant des différences entre races pures trouvées par MoLENAT et al. (I974) et SEIIIER

\section{TABLEAU 3}

Valeur initiale de la marge unitaire (en francs) pour les différents types génétiques Initial value of the gross profit per slaughter pig (in francs) in the different breeding groups

\begin{tabular}{|c|c|c|c|c|c|c|c|c|c|c|}
\hline \multirow[t]{2}{*}{$\begin{array}{l}\text { Types génétiques } \\
\text { (breeding groups) }\end{array}$} & \multicolumn{4}{|c|}{$\begin{array}{l}\text { Races pures }\left(\mathrm{G}_{\mathrm{y}, o}\right) \\
(\text { purebreds })\end{array}$} & \multicolumn{6}{|c|}{$\begin{array}{c}\text { Croisements }\left(\mathrm{G}_{y, o}+\mathrm{E}_{\mathrm{y}}\right) \\
(\text { crossbreds })\end{array}$} \\
\hline & LW & $\mathrm{LF}$ & LB & $\mathrm{P}$ & $\begin{array}{l}\mathrm{LW} \\
\times \mathrm{LF}\end{array}$ & $\begin{array}{l}\mathrm{LF} \\
\times \mathrm{LW}\end{array}$ & $\begin{array}{l}\mathrm{LW} \\
\times \quad \mathrm{F}_{1}\end{array}$ & $\begin{array}{l}\mathrm{LF} \\
\times \quad \mathrm{F}_{1}\end{array}$ & $\begin{array}{l}\mathrm{LB} \\
\times \quad \mathrm{F}_{1}\end{array}$ & $\begin{array}{ll}\mathrm{P} & \\
\times & \mathrm{F}_{1}\end{array}$ \\
\hline $\begin{array}{l}\text { Marge unitaire } . \dot{ } \\
\text { (gross profit per pig) }\end{array}$ & 130,0 & I 25,8 & I 54,8 & I 25,0 & I 34,2 & I 34,2 & I 34,3 & I 30,0 & I 45,5 & 134,0 \\
\hline
\end{tabular}


et al. (r974) et en faisant des hypothèses sur les effets d'hétérosis, compte tenu des estimations disponibles dans la littérature (SELIIER, I976b). Nous en avons déduit les valeurs initiales $\mathrm{G}_{\mathbf{y}}$, o et $\mathrm{E}_{\mathrm{y}}$ (tabl. 3), en supposant qu'il n'y a pas d'effet de l'âge de la mère sur la marge unitaire. Dans le cas des mâles entiers vendus à roo $\mathrm{kg}$, cette marge unitaire qui, rappelons-le, a été supposée indépendante du type génétique et non modifiée par la sélection, a été prise égale à $30 \mathrm{~F}$.

\section{c) Supériorité des animaux retenus après sélection}

La sélection s'effectue dans les noyaux de race pure qui, à la fois, assurent leur propre renouvellement en mâles et en femelles et fournissent les femelles de renouvellement aux troupeaux de multiplication et les mâles de renouvellement aux troupeaux de multiplication et de production. D'une façon générale, les supériorités des animaux retenus après sélection dans la cohorte $i$ dépendent des intensités de sélection $\Delta \mathrm{X}_{i}$ et $\Delta \mathrm{Y}_{i}$ relatives respectivement à 1'indice de sélection sur la prolificité $(\mathrm{X})$ et à l'indice de sélection sur la performance de production ( $\mathrm{Y}$ ) et des écarts-type $\sigma_{\mathbf{x}}$ et $\sigma_{\mathbf{y}}$ de ces indices.

\section{TABLEAU 4}

Intensités de sélection appliquées aux reproducteurs issus des noyaux de race pure Intensities of selection applied to breeding animals born in nucleus herds

\begin{tabular}{|c|c|c|c|c|}
\hline \multicolumn{3}{|c|}{$\begin{array}{l}\text { Type de reproducteur } \\
\text { (type of breeding animal) }\end{array}$} & \multicolumn{2}{|c|}{$\begin{array}{l}\text { Intensités de sélection } \\
\text { (intensities of selection) }\end{array}$} \\
\hline $\begin{array}{c}\text { noyau } \\
\text { (nucleus) }\end{array}$ & $\begin{array}{l}\text { sexe } \\
(\text { sex })\end{array}$ & $\begin{array}{l}\text { troupeaux d'utilisation } \\
\text { (herds of utilization) }\end{array}$ & $\begin{array}{l}\text { prolificité }(\Delta \mathrm{X} i) \\
\quad \text { (prolificacy) }\end{array}$ & $\begin{array}{c}\text { indice de production } \\
\left(\Delta \mathrm{Y}_{1}\right) \\
\text { (production index })\end{array}$ \\
\hline \multirow{4}{*}{ LW et $L F$} & \multirow{2}{*}{ q } & sélection . . . . . & $i\left(r_{1}\right) \S$ & $\mathrm{i}\left(p_{1}\right)$ \\
\hline & & multiplication & $\frac{\lambda_{1}}{\lambda_{1}-I} \mathrm{i}\left(v_{1}\right)$ & $\frac{\lambda_{1}}{\lambda_{1}-\mathrm{I}} \mathrm{i}\left(p_{1}\right)$ \\
\hline & \multirow[b]{2}{*}{$\sigma$} & sélection . . . . & $\mathbf{i}\left(r_{2}\right)$ & $\mathbf{i}\left(p_{2}\right)$ \\
\hline & & $\begin{array}{l}\text { multiplication et pro- } \\
\text { duction . . . . }\end{array}$ & $\mathrm{i}\left(r_{3}\right)$ & $\frac{p_{3} \mathrm{i}\left(p_{3}\right)-p_{2} \mathrm{i}\left(p_{2}\right)}{p_{3}-p_{2}}$ \\
\hline \multirow{3}{*}{$\mathrm{T} \dagger$} & 우 & sélection . . . . . . & o & $i\left(\lambda_{1}\right)$ \\
\hline & \multirow[b]{2}{*}{$\sigma$} & sélection $\cdot \cdot \ldots \cdot \cdot$ & o & $\mathrm{i}\left(\lambda_{2}\right)$ \\
\hline & & multiplication $\ldots$ & o & $\frac{\lambda_{2}}{\lambda_{2}-I} i\left(\lambda_{2}\right)$ \\
\hline
\end{tabular}

$\S \mathrm{i}\left(r_{1}\right)$ est l'intensité de sélection calculée d'après la loi normale pour le taux $r_{1}\left(\mathrm{i}\left(r_{1}\right)=z / r_{1}\right.$ assuming a normal distribution).

$\dagger \mathrm{I}=$ Piétrain, Landrace Belge ou Large White "viande " (Pietrain, Belgian Irandrace or Large White sire strain). 

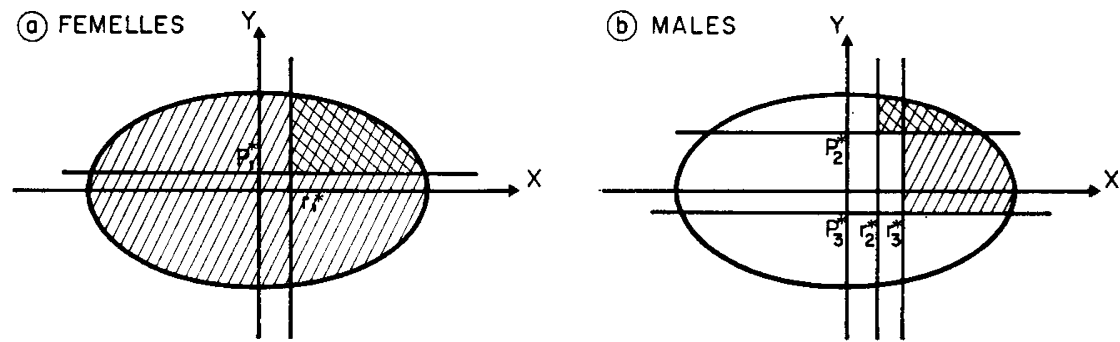

FIG. 2. - Modes de choix des reproducteurs dans les noyaux "femelles" Procedures for selection of breeding animals in the "female " nucleus herds $\mathrm{X}$ : indice de sélection sur la prolificité (selection index on litter size) $\mathrm{Y}$ : indice de sélection sur la performance de production (selection index on production performance) hachures doubles : animaux destinés au renouvellement des noyaux de sélection. (double hachures : animals retained for replacement of nucleus herds).

hachures simples : animaux destinés au renouvellement de l'étage de multiplication (simple hachures: animals moved for replacement to multiplier herds) $r^{*}{ }_{1}$ est l'abscisse du point de troncature correspondant au taux de sélection $r_{1}$ $\left(r^{*}{ }_{1}\right.$ is the abscissa of the point of truncation for the selection rate $\left.r_{1}\right)$.

\section{a. Intensités de sélection (tabl. 4)}

Les effectifs des noyaux des races Large White et Landrace étant ajustés aux besoins en femelles, toutes les jeunes femelles sont retenues pour le renouvellement, les meilleures restant dans les noyaux et les moins bonnes étant transférées dans les troupeaux de multiplication.

Une première sélection est effectuée sur l'indice de prolificité $\mathrm{X}$ (avec un taux $r_{1}$ ). Les animaux sélectionnés sont alors contrôlés sur leurs propres performances de croissance et d'adiposité et la fraction $p_{1}$ supérieure est définitivement retenue pour le renouvellement des noyaux de races pures (fig. $2 a$ ). Les taux $r_{1}$ et $p_{1}$ sont liés par la relation $r_{1} p_{1}=\lambda_{1}$ où $\lambda_{1}$ est le rapport du nombre de femelles nécessaires au renouvellement du noyau au nombre de candidates. On peut en déduire les différentielles de sélection pour les femelles conservées dans les noyaux de race pure et pour les femelles assurant le renouvellement des troupeaux de multiplication.

Le mode de choix des mâles de renouvellement des troupeaux de sélection est identique à celui des femelles, avec des taux $r_{2}$ et $p_{2}$. On a par ailleurs supposé que les mâles destinés aux étages inférieurs sont choisis, avec des taux $r_{3}$ et $p_{3}$, parmi les animaux contrôlés sur leur performance de production, ce qui implique $r_{3}<r_{2}$ (fig. $2 b$ ). Ces taux sont liés par les relations $r_{2} p_{2}=\lambda_{2}$ et $r_{3}\left(p_{3}-p_{2}\right)=\lambda_{3}, \lambda_{3}$ étant ici le rapport du nombre de mâles nécessaires pour le renouvellement des étages inférieurs au nombre de candidats.

Dans le cas de la souche mâle spécialisée, l'effectif du noyau de sélection est fixé par le besoin en mâles de renouvellement de l'étage de production. Aucune sélection n'est faite sur la prolificité $\left(r_{1}=r_{2}=r_{3}=\mathrm{r}\right)$ et on a les relations $p_{1}=$ $\lambda_{1}$ et $p_{2}=\lambda_{2}$.

\section{ß. Écarts-type des indices de sélection}

En ce qui concerne la sélection des performances de croissance et de carcasse, les caractères mesurés sur les candidats à la sélection sont la durée d'engraissement de 25 à Ioo $\mathrm{kg}\left(\mathrm{P}_{1}\right)$, les épaisseurs de lard au rein $\left(\mathrm{P}_{2}\right)$ et au dos $\left(\mathrm{P}_{3}\right)$ au poids 
de $100 \mathrm{~kg}$. La valeur génétique globale $\mathrm{H}$, qui a la signification d'une marge unitaire (prix de vente - coût de l'engraissement), s'inspire de celle décrite par OL,IVIER (I97I) :

$\mathrm{H}=-0,8 h_{1}-0,52 h_{2}+\mathrm{I}, 5 h_{3}+2 h_{4}-8 h_{5}-7 h_{6}-\mathrm{I}, \mathrm{I} h_{7}-\mathrm{I}, \mathrm{I} h_{8}$

où $h_{1}, h_{2}, \ldots, h_{8}$ sont les valeurs génétiques additives respectivement pour la consommation d'aliment $(\mathrm{kg})$, la durée d'engraissement $(\mathrm{j})$, jles poids de jambon, longe, bardière et panne par demi-carcasse $(\mathrm{kg})$, les épaisseurs de lard au rein et au dos $(\mathrm{mm})$. Les variances et covariances estimées par OLLIVIER (I970) ont été utilisées pour calculer les coefficients de l'indice $\mathrm{Y}\left(\mathrm{Y}=-0,756 \mathrm{P}_{1}-\mathrm{I}, 977 \mathrm{P}_{\mathbf{2}}-\mathrm{I}, 092 \mathrm{P}_{3}\right)$, son écart-type $\left(\sigma_{y}=I_{5}, 04\right)$ et sa corrélation avec $\mathrm{H}\left(\rho_{\mathbf{y}}=0,768\right)$. Nous n'avons pas envisagé l'introduction de l'indice de consommation comme variable prédictrice de $\mathrm{H}$, car elle n'améliore la corrélation $p_{y}$ que de $4 \mathrm{p}$. Ioo.

En ce qui concerne la prolificité, la valeur génétique des candidats à la sélection dans les noyaux "femelles" peut être estimée de quatre façons différentes selon que l'information utilisée concerne seulement la mère dı candidat ou, en plus de la mère, un certain nombre d'apparentées contemporaines de la mère (tantes et demi-tantes) et selon que chacune de ces femelles est connue sur une ou deux portées. Dans le cas où l'information utilisée ne concerne pas seulement la mère,

TABLEAU 5

Estimation de la supériorité génétique pour la prolificité dans les quatre situations de sélection (I) Estimation of the genetic superiority for litter size in the four selection methods

\begin{tabular}{|c|c|c|c|c|}
\hline $\begin{array}{l}\text { Apparentées prises en compte } \\
\text { (velatives taken in account) }\end{array}$ & \multicolumn{2}{|c|}{$\begin{array}{l}\text { Mère } \\
(\text { dam })\end{array}$} & \multicolumn{2}{|c|}{$\begin{array}{l}\text { Mère }+ \text { tantes } \\
\text { et demi-tantes } \\
(\text { dam }+ \text { aunts } \\
\text { and half-aunts })\end{array}$} \\
\hline $\begin{array}{l}\text { Nb. de portées / truie. . . . . . . . . . } \\
\text { (no. of litters / sow) }\end{array}$ & I & 2 & I & 2 \\
\hline $\begin{array}{l}\text { Corrélation entre l'indice de sélection } \\
\text { et la valeur génétique }\left(\rho_{\mathrm{x}}\right) . . .5 \\
\text { (correlation between selection index and } \\
\text { breeding value) }\end{array}$ & 0,158 & 0,209 & 0,228 & 0,289 \\
\hline $\begin{array}{l}\text { Écart-type de l'indice de sélection }\left(\sigma_{\mathrm{x}}\right) \\
\text { (standard deviation of the selertion in- } \\
\quad \text { dex) }\end{array}$ & 0,130 & 0,172 & $0, \mathbf{1} 87$ & 0,236 \\
\hline 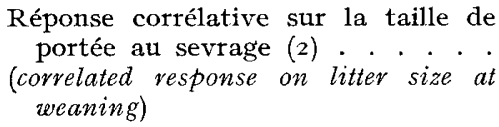 & 0,083 & 0,109 & $0, \mathbf{I}+9$ & $0,15 \mathrm{I}$ \\
\hline
\end{tabular}

(1) Hypothèses sur les paramètres (Assumed values of the parameters).

TPN : taille de portée à la naissance (litter size at birth).

TPS : taille de portée au sevrage (litter size at weaning).

Héritabilité de TPN et TPS et répétabilité de TPN : respectivement o, 10, o,08 et o, 15; corrélation génétique entre TPN et TPS : 0,80; écart-type phénotypique de TPN et TPS : respectivement 2,604 et 2,315 .

(') Pour une différentielle de sélection égale à un écart-type de l'indice (For a selection differential equa! to one standard deviation of the index). 
TABLEAU 6

Paramètres démographiques

Demographic parameters

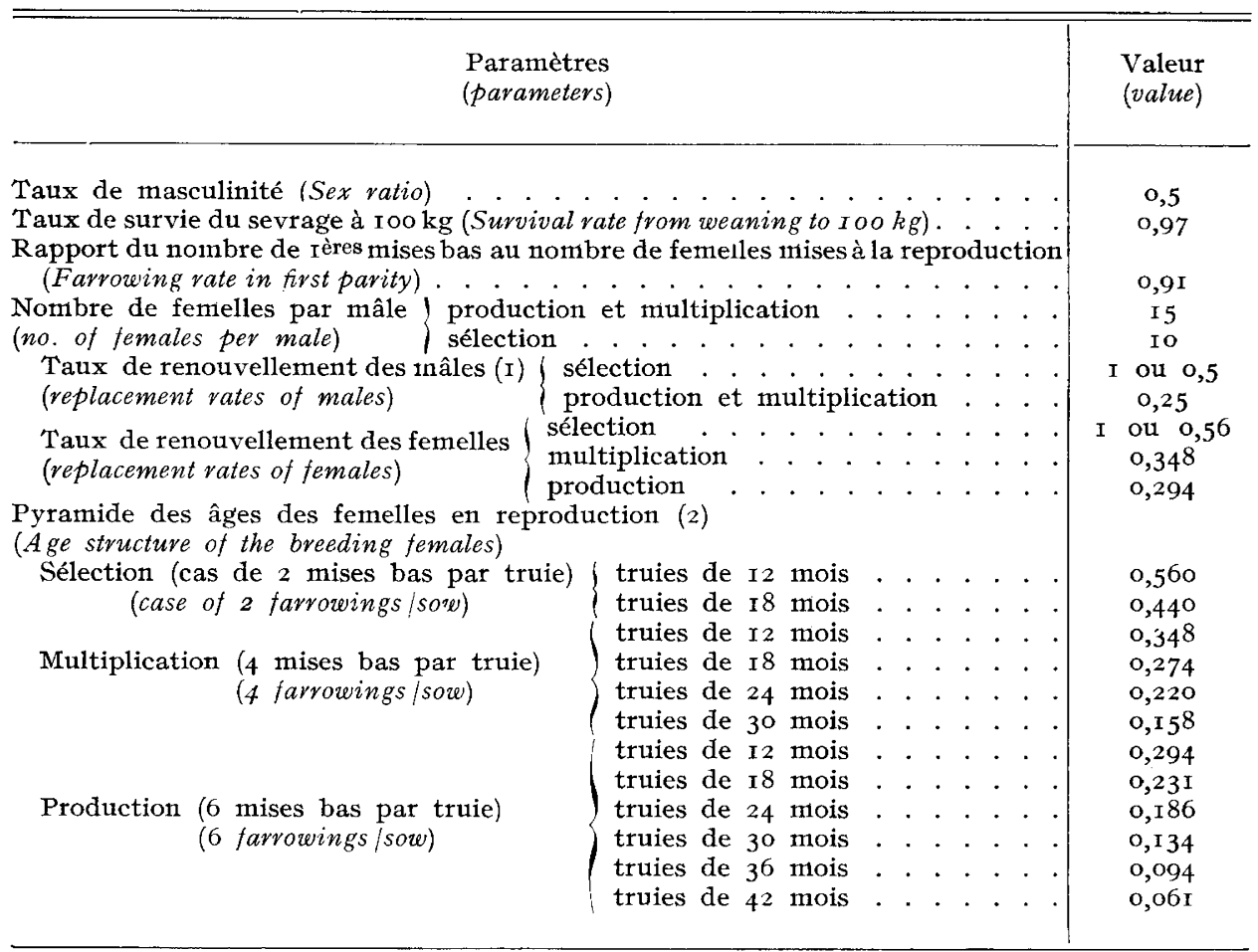

(1) On a fait l'hypothèse qu'aucun mâle n'était réformé d'une période de saillie à la suivante (It has been assumed that no male was culled from one mating period to the following one).

$\left({ }^{2}\right)$ D'après les données de LEGaulT et al. (1975) (From data of LEGAult et al., I975).

nous avons supposé qu'étaient connues les performances de la mère, d'une propre sœur et de neuf demi-sœurs paternelles du père, de neuf demi-sœurs paternelles de la mère : ce volume d'information correspond à la situation moyenne pour chaque candidat, compte tenu du niveau de prolificité du Large White et du Landrace Français et du nombre moyen de filles conservées pour la reproduction par verrat et par truie. Dans tous les cas, le critère de sélection concerne la taille de portée à la naissance et la réponse corrélative sur la taille de portée au sevrage a été calculée. Les paramètres phénotypiques et génétiques utilisés pour ces calculs qui conduisent aux résultats du tableau 5 proviennent des études de LEGaulit $($ I970 $b$ ) et STRANG et KING (I970).

\section{3. - Estimation des effectifs de femelles et du nombre de reproducteurs réformés}

Avec 1'hypothèse de constance du nombre de reproducteurs dans le temps et en supposant un effectif de Ioo ooo femelles $F_{1}$ dans les élevages de production, on peut, à partir des valeurs des prolificités, des taux de survie, du rapport du 
nombre de mâles au nombre de femelles en reproduction, estimer les différents effectifs de mères et d'animaux réformés intervenant dans les schémas. Dans tous les cas, on a supposé que les femelles $\mathrm{F}_{1}$ sont également réparties entre les deux croisements réciproques (50 000 femelles de père Large White et 50000 femelles de père Landrace) et, pour le croisement en retour, sont accouplées à parts égales aux deux races de mâles.

Les paramètres démographiques nécessaires figurent aux tableaux I et 6 . On a supposé que les femelles mettent bas pour la première fois à l'âge d'un an puis tous les 6 mois.

Cet ensemble de paramètres permet de calculer les effectifs de reproducteurs dont une partie est donnée sur les figures $\mathrm{I} a$ et $\mathrm{I} b$. Ces paramètres permettent également de calculer les coefficients $\lambda_{1}, \lambda_{2}$ et $\lambda_{3}$ qui lient les taux de sélection.

\section{4. - Expression algébrique des recettes}

Les recettes sont calculées à partir de leur expression générale :

avec $\mathrm{R}_{1}=y_{t} \mathrm{~N}^{\prime}{ }_{t}$

$$
\mathrm{R}=\sum_{t=1}^{T}\left(\mathrm{R}_{1} t-\mathrm{R}_{2} t\right) \theta^{t}
$$

$$
\mathbf{R}_{2}=y_{t} \mathrm{~A}^{\prime}
$$

où : $x_{t}$ et $y_{t}$ sont les vecteurs de dimension $n$ des prolificités et des marges unitaires donnés par les relations (7) et (8).

A est le vecteur de dimension $n$ des effectifs de reproducteurs retenus pour le renouvellement.

$x^{\prime}{ }_{t}$ et $\mathrm{A}^{\prime}$ sont les transposés de $x_{t}$ et $\mathrm{A}$.

$\mathrm{N}$ est la matrice $n \times n$ des effectifs de mères, "corrigés" pour le taux de survie $k$.

Ces expressions peuvent s'écrire :

$\mathbf{R}_{\mathbf{1}}=\left(y_{o}+\Delta \mathrm{G}_{\mathbf{y}} \mathfrak{D}_{t}\right) \mathbf{N}\left(x^{\prime}{ }_{o}+\mathfrak{D}^{\prime}{ }_{t} \Delta \mathrm{G}^{\prime}{ }_{\mathbf{x}}\right)$

$\mathbf{R}_{2}=\left(y_{0}+\Delta \mathrm{G}_{\mathbf{y}} \mathfrak{D}_{t}\right) \mathbf{A}^{\prime}$

On peut alors décomposer les recettes totales en :

(9) $\mathrm{R}=\alpha+\Delta \mathrm{G}_{\mathbf{y}} \beta+\gamma \Delta \mathrm{G}_{\mathbf{x}}^{\prime}+\Delta \mathrm{G}_{\mathbf{y}} \delta \Delta \mathrm{G}^{\prime}{ }_{\mathbf{x}}$

avec $\alpha=\sum_{t=\mathrm{r}}^{T} \theta^{t}\left(y_{0} \mathrm{~N} x_{0}^{\prime}-y_{o} \mathrm{~A}^{\prime}\right)$

$$
\begin{aligned}
& \beta=\sum_{t=1}^{T} \theta^{t} \mathbb{D}_{t}\left(\mathrm{~N} x_{o}^{\prime}-\mathrm{A}^{\prime}\right) \\
& \gamma=\sum_{t=1}^{T} \theta^{t}\left(y_{o} \mathrm{ND}^{\prime}{ }_{t}\right) \\
& \delta=\sum_{t=1}^{T} \mathcal{D}_{t} \mathrm{NDD}_{t} \theta^{t}
\end{aligned}
$$

Rappelons que, dans le cas du croisement triple, les recettes des trois premiers semestres ont été calculées séparément.

La décomposition des recettes, telle qu'elle apparaît dans la relation (9), permet d'individualiser :

- une part due au seul croisement $(\alpha)$; 
- une part due à la sélection sur les caractères de croissance et de carcasse $\left(\Delta \mathrm{G}_{\mathbf{y}} \beta\right)$;

- une part due à la sélection sur la prolificité $\left(\gamma \Delta \mathbf{G}^{\prime}{ }_{\mathbf{x}}\right)$;

- une part due à $l^{\prime}$ " effet conjoint " des deux types de sélection $\left(\Delta \mathrm{G}_{\mathbf{y}} \delta \Delta \mathrm{G}^{\prime} \mathbf{x}\right)$.

\section{C. - Estimation des coûts}

Partant de la situation initiale définie plus haut, nous avons tenté d'estimer les coûts engagés en nous intéressant particulièrement à la fraction des coûts qui varient d'un schéma à l'autre. Les coûts peuvent ainsi être subdivisés en coûts fixes, coûts dépendant des effectifs d'animaux et coûts liés à la sélection proprement dite.

\section{Coûts fixes}

Il est utile de rappeler ici que nous avons fait l'hypothèse que les effectifs de reproducteurs dans les étages de multiplication et de production sont les mêmes quel que soit le schéma : les frais d'entretien de ces reproducteurs représentent donc đes coûts fixes (notés $\psi$ ) et ont été décomposés en coûts alimentaires et coûts autres qu'alimentaires. Pour les truies, nous avons supposé une consommation journalière d'aliment et un prix de l'aliment de $2,5 \mathrm{~kg}$ et $0,80 \mathrm{~F} / \mathrm{kg}$ pour les périodes d'attente de saillie et de gestation, de $5 \mathrm{~kg}$ et $0,85 \mathrm{~F} / \mathrm{kg}$ pour la période de lactation; les coûts autres qu'alimentaires ont été estimés à $2 \mathrm{~F} /$ jour. Les coûts d'entretien des verrats reproducteurs ont été calculés de la même façon en supposant une consommation moyenne journalière d'aliment de $3,5 \mathrm{~kg}$ et un prix de l'aliment de $0,80 \mathrm{~F} / \mathrm{kg}$. De ces coûts ont été retranchées les valeurs de réforme des reproducteurs, supposées indépendantes du type génétique et de 1'âge de l'animal, constantes dans le temps et égales à $550 \mathrm{~F}$ pour les truies et $300 \mathrm{~F}$ pour les verrats.

\section{Cô̂ts proportionnels aux effectifs}

Ces coûts, notés $\varepsilon$, ont trois origines :

a. investissement en bâtiments : 1'année o de la période d'étude est définie en particulier par le début des opérations de sélection, ce qui nécessite la construction de bâtiments pour les contrôles de performance. A partir de données relatives à la station de sélection du Rheu, nous avons supposé que ces bâtiments représentaient, dans chaque noyau de sélection, un investissement de 8 ooo $\mathrm{F}$ par femelle reproductrice. En principe, il conviendrait, dans l'estimation du bénéfice, de soustraire de cet investissement sa valeur de déclassement actualisée à 1'horizon final; en pratique, faute de données, cette valeur a été négligée.

b. investissement en animaux : il est supposé, rappelons-le, que dans son état initial, la structure démographique de la population est celle du croisement en retour. La mise en place du croisement triple nécessite la création d'un nouveau noyau de sélection. Dans le cas du croisement triple, l'investissement en animaux, supposé engagé en totalité à 1'année o, a été estimé sur la base de I ooo F/femelle et de I $500 \mathrm{~F} / \mathrm{mâle}$. 
c. coûts d'entretien des reproducteurs des noyaux de sélection. Ils ont été calculés de la même façon que pour les reproducteurs des autres étages. Ce coût est fonction de l'effectif total des reproducteurs de l'étage de sélection (existence ou non d'un noyau "mâle ") et, dans le cas des noyaux "femelles ", des taux de renouvellement pratiqués.

Les coûts proportionnels aux effectifs ont donc la forme générale

$$
\varepsilon=\sum_{j \in J^{\prime}} \mathrm{N}_{j}\left(\dot{\eta}_{j}+\sum_{t=\mathrm{I}}^{T} \theta^{t} \varepsilon_{j}\right)
$$

où $\mathrm{J}^{\prime}$ est l'ensemble des indices de cohortes de femelles en reproduction dans l'étage de sélection et $\mathrm{N}_{j}$ le nombre de femelles de la cohorte $j$ et où $\varepsilon_{j}$ et $\dot{\eta}_{j}$ sont respectivement le coût d'entretien et l'investissement initial (bâtiments, animaux), exprimés par femelle.

\section{3. - Coûts liés à la sélection}

Nous entendons par là les coûts du contrôle de performances des candidats à la sélection dans les noyaux. Nous avons supposé que les informations servant au calcul de l'indice de sélection sur la prolificité sont disponibles sans frais supplémentaires. Les coûts du contrôle de croissance et d'adiposité (pesées, mesures d'épaisseur de lard) ont été estimés à $9 \mathrm{~F}$ par animal, les animaux éliminés par la sélection sur la prolificité n'étant pas soumis au contrôle individuel. Les coûts totaux annuels de contrôle dans le noyau de sélection $l-$ Large White $(l=\mathrm{I})$, Landrace Français $(l=2)$ ou souche mâle $(l=3)$ - sont de la forme :

$$
9 \sum_{m} \mathrm{~N}_{m l} \frac{x_{m l, o}}{2}\left(r_{1 l}+r_{2 l}\right)
$$

où $r_{1}$ et $r_{2}$ sont les taux de sélection sur la prolificité respectivement chez les mâles et chez les femelles,

$\mathrm{N}_{m l} \quad$ est le nombre de femelles donnant la $m^{\text {ième }}$ mise bas dans le noyau $l(m=\mathrm{I}$ ou 2$)$,

$x_{m l, o} \quad$ est la prolificité initiale de ces femelles (on a négligé l'accroissement possible des différentielles de sélection dû à l'augmentation de la taille de portée).

Dès lors, les coûts liés à la sélection s'écrivent :

$$
\sum_{l} \varphi_{l}\left(r_{1 l}+r_{2 l}\right) / 2
$$

avec

$$
\varphi_{l}=9 \sum_{t=\mathrm{I}}^{T} \theta^{t}\left(\sum_{m} \mathrm{~N}_{m l} x_{m l, o}\right)
$$

Notons que dans le cas du noyau "mâle " $(l=3), r_{1 l}=r_{2 l}=\mathrm{I}$ et $m=$ I. L,es coûts liés à la sélection s'écrivent finalement :

$$
\varphi=\varphi_{I}\left(r_{1 I}+r_{2 I}\right) / 2+\varphi_{2}\left(r_{12}+r_{22}\right) / 2+\varphi_{3}
$$

\section{D. - Détermination du schéma d'amélioration génétique optimum}

Le bénéfice actualisé prend donc la forme :

$$
\mathrm{B}=\mathrm{R}-\mathrm{C}=\left(\alpha+\Delta \mathrm{G}_{\mathbf{y}} \beta+\gamma \Delta \mathrm{G}^{\prime} \mathbf{x}+\Delta \mathrm{G}_{\mathbf{y}} \delta \Delta \mathrm{G}_{\mathbf{x}}^{\prime}\right)-(\dot{\psi}+\varepsilon+\varphi)
$$


Les paramètres $\alpha, \beta, \gamma, \delta, \varepsilon, \varphi$ sont calculés pour chacune des situations étudiées; on cherche alors les valeurs des taux de sélection maximisant $B$, compte tenu des contraintes suivantes :

$\lambda_{1 l}<r_{1 l}<\mathrm{I}$

$\lambda_{2 l}<r_{2 l}<\mathrm{I}$

$\lambda_{3 l}<r_{3 l}<r_{2 l}$

$$
\begin{aligned}
& r_{1} l p_{1} l=\lambda_{1} l \\
& r_{2 l} p_{2 l}=\lambda_{2} l \\
& r_{3}\left(p_{3}-p_{2}\right)=\lambda_{3} l
\end{aligned}
$$

$\lambda_{1 l}<p_{1} l<\mathrm{I}$

$\lambda_{2 l}<p_{2 l}<\mathrm{I}$

$p_{2 l}<p_{3 l}<\mathrm{I}$

Par élimination de variables, ce problème est ramené à 6 variables $\left(r_{1 r}, r_{2 I}\right.$, $\left.r_{3 x}, r_{12}, r_{22}, r_{32}\right)$ et I4 contraintes.

Pour déterminer le maximum, un programme d'optimisation sous contraintes utilisant la méthode du gradient réduit (FAURE et HUARD, 1965) a été écrit. Cette méthode, proche de la programmation linéaire, consiste à rechercher le maximum d'une fonction en partant d'un point initial réalisable (satisfaisant aux contraintes) et en faisant varier des variables dites de base dans la direction assurant l'augmentation maximale de la fonction. Dès que le maximum local de la fonction est atteint, une nouvelle direction est calculée et les nouvelles valeurs optimales des variables de base sont recherchées. Ce cheminement est interrompu par des changements de base éventuels si les variables hors base deviennent irréalisables et se termine lorsqu'en un point donné, la fonction ne peut être améliorée dans aucune direction.

\section{III. - Résultats}

Pour faciliter l'exposé des résultats, nous avons choisi de les présenter en montrant séparément l'influence des 5 facteurs de variation envisagés ici sur le " gain dû au croisement " d'une part, le "gain dû à la sélection " d'autre part, avant de comparer les schémas d'un point de vue global. Par "gain dû au croisement" et "gain dû à la sélection", nous désignons respectivement les quantités $\mathrm{B}_{c}=$ $\alpha-\dot{\psi}-\varepsilon$ et $\mathrm{B}_{s}=\Delta \mathrm{G}_{y} \beta+\gamma \Delta \mathrm{G}^{\prime}{ }_{x}+\Delta \mathrm{G}_{y} \delta \Delta \mathrm{G}^{\prime}{ }_{x}-\varphi$ de la relation (Io). Cette décomposition comporte une part d'arbitraire : en particulier, les termes $\beta, \gamma$ et $\delta$ qui interviennent dans l'expression du gain dû̀ à la sélection dépendent du plan de croisement pratiqué et par ailleurs, certains éléments du cô̂t sont attribués à l'un ou l'autre des gains alors qu'ils leur sont communs dans une certaine mesure; ces éléments de coût étant petits vis-à-vis des recettes, ce dernier point n'a cependant pas une grande importance sur le plan pratique.

\section{A. - Influence des facteurs de variation sur le gain dîu au croisement}

\section{Taux de renouvellement dans les noyaux "femelles":}

Vis-à-vis de $\mathbf{B}_{c}$, la seule influence des taux de renouvellement pratiqués dans les noyaux "femelles " s'exerce par le biais de l'effectif et de 1'âge moyen des reproducteurs de ces noyaux. Cet effectif étant très faible relativement au nombre total de reproducteurs de l'ensemble de la population (à peine I p. Ioo), les taux de 
renouvellement n'ont pas d'effet notable sur les recettes dues au croisement $(\alpha)$. Bien que l'influence de ces taux sur les coûts proportionnels aux effectifs $(\varepsilon)$ soit importante (en particulier du point de vue des coûts d'entretien des reproducteurs des noyaux de sélection), le gain dû au croisement est très peu affecté.

\section{Conditions d'actualisation:}

L'influence de la durée et du taux d'actualisation sur le gain dû au croisement a été étudiée seulement pour le croisement en retour et pour le croisement triple avec le Piétrain. Dans le cas du croisement en retour, pour lequel l'équilibre démographique est supposé réalisé dès l'instant initial, le gain $B_{c}$ non actualisé croît linéairement avec le temps. Dans le cas du croisement triple, en dépit de l'existence d'une phase de mise en place, la croissance de $\mathbf{B}_{c}$ non actualisé ne s'écarte pas sensiblement de la linéarité. L'actualisation diminue fortement cette croissance puisque, sur 30 semestres, la valeur de $B_{c}$ actualisé au taux de $20 \mathrm{p}$. roo est à peine le tiers de $\mathrm{B}_{c}$ non actualisé. Les bénéfices dus au croisement atteignent des valeurs considérables au bout /de 30 semestres (environ I,5 milliard de $\mathrm{F}$ non actualisés) mais il faut remarquer que ce sont des bénéfices cumulés.

\section{Plans de croisement:}

Le classement des différents plans de croisement et même leur mérite relatif (tabl. 7) sont pratiquement indépendants des conditions d'actualisation et des taux de renouvellement. Du point de vue du gain dû au croisement, le croisement triple avec le Landrace Belge apparaît comme le plan le plus efficace, suivi de près par le croisement triple avec la souche Large White "viande ". Le croisement en retour et le croisement triple avec le Piétrain donnent des résultats inférieurs de I5 à 20 p. roo.

\section{TABLEAU 7}

Influence du plan de croisement sur le gain dî au croisement $B_{c}$ ( $\mathrm{ooo}=$ meilleur plan de croisement)

Influence of the crossbreeding plan on the gain due to crossing $B_{c}$ $($ I $00=$ best crossbreeding plan)

\begin{tabular}{|c|c|c|c|c|}
\hline \multirow{2}{*}{$\begin{array}{l}\text { Plan de croisement } \\
\text { (crossbreeding plan) }\end{array}$} & \multicolumn{3}{|c|}{$\begin{array}{l}\text { Croisement triple avec } \\
\text { (three-way cross with) }\end{array}$} & \multirow{2}{*}{$\begin{array}{l}\text { Croisement } \\
\text { en retour } \\
\text { (backcross) }\end{array}$} \\
\hline & $\begin{array}{c}\text { Landrace Belge } \\
\text { (Belgian } \\
\text { Landrace) }\end{array}$ & $\begin{array}{l}\text { Large White } \\
\text { " viande" } \\
\text { (Large White } \\
\text { sire strain) }\end{array}$ & $\begin{array}{l}\text { Piétrain } \\
\text { (Pietrain) }\end{array}$ & \\
\hline $\mathrm{B}_{c}(\mathrm{I}) \ldots \ldots$ & Ioo & $95, \mathrm{I}$ & $8 \mathrm{r}, \mathrm{o}$ & 85,5 \\
\hline
\end{tabular}

${ }^{(1)}$ Les valeurs de $B_{c}$ données dans ce tableau correspondent à une durée d'actualisation de 30 semestres et à un taux d'actualisation de o p. Ioo (For a discounting period of 30 half-years and a discounting rate of o p. IOO). 
Il est évident que ces résultats sont la conséquence directe des hypothèses faites sur la valeur initiale des races paternelles pour la performance de production et pour l'effet sur la taille de portée. La forte supériorité des croisés Landrace Belge pour les caractères de croissance et de carcasse est en partie compensée, vis-à-vis du croisement triple avec le mâle Large White "viande ", par l'effet favorable de ce dernier sur la taille de la portée à la naissance. Par contre, dans le cas du croisement triple avec le Piétrain, dont les descendants croisés ont la même valeur que ceux d'un mâle Large White et pour lequel les tailles de portée sont identiques à celles du croisement triple avec le Landrace Belge, les recettes sont nettement inférieures. Enfin, on remarquera que le croisement triple n'est pas systématiquement le meilleur plan puisque le croisement en retour donne de meilleurs résultats que l'utilisation du Piétrain comme lignée mâle du croisement terminal, avec les valeurs des paramètres qui ont été utilisées ici.

\section{B. - Influence des facteurs de variation sur le gain dî̀ à la sélection}

Les facteurs de variation étudiés influençant non seulement la valeur du gain dû à la sélection mais aussi les taux de sélection optimaux à pratiquer, nous présenterons pour chacun de ces facteurs leur influence sur ces deux groupes de variables.

On se souviendra qu'après réduction du nombre de variables, les taux de sélection envisagés sont les taux de sélection sur la prolificité des femelles $\left(r_{1 x}\right.$ et $\left.r_{12}\right)$ et des mâles $\left(r_{21}\right.$ et $\left.r_{2^{2}}\right)$ destinés au renouvellement des deux noyaux "femelles" (respectivement Large White et Landrace Français), ainsi que les taux de sélection sur la prolificité des mâles issus de ces mêmes noyaux et destinés au renouvellement des étages inférieurs $\left(r_{3^{x}}\right.$ pour les mâles Large White, $r_{3^{2}}$ pour les mâles Landrace Français).

Dans tous les cas étudiés, les taux optimaux sont identiques dans les deux noyaux $\left(r_{1 I}=r_{12}=r_{1}, r_{2 I}=r_{22}=r_{2}, r_{3 x}=r_{3^{2}}=r_{3}\right)$. Par la suite, nous présenterons donc les taux correspondants aux reproducteurs issus d'un seul des deux noyaux.

Enfin, rappelons que seuls le croisement en retour et le croisement triple avec le Piétrain ont été analysés de façon complète. Pour les deux autres croisements triples, nous nous sommes limités à une durée d'actualisation de 30 semestres et au taux de renouvellement des mâles maximum, ces valeurs apparaissant comme les plus intéressantes au vu des résultats obtenus avec les deux premiers plans.

\section{Conditions d'actualisation}

\section{a) Effet sur les taux de sélection}

De façon générale, les taux de sélection optimaux sur la prolificité décroissent quand la durée d'actualisation augmente ou quand le taux d'actualisation diminue. Dans le cas du croisement en retour, ces taux de sélection ne s'écartent de roo p. Ioo que lorsque le bénéfice est estimé sur 30 semestres, avec un taux d'actualisation inférieur à $20 \mathrm{p}$. Ioo. Nous donnons dans le tableau 8 les valeurs optimales de ces taux dans le cas du croisement triple avec le Piétrain. On notera que l'effet de la durée d'actualisation sur le taux de sélection des femelles $\left(r_{1}\right)$ 
n'obéit pas à la règle énoncée plus haut; en effet le taux $r_{1}$ est maximum pour une durée d'actualisation de 20 semestres, dans toutes les situations; d'autre part, contrairement à ce qu'on observe pour les mâles $\left(r_{2}\right.$ et $\left.r_{3}\right)$, ce taux varie peu avec la durée.

\section{TABLEAU 8}

Taux de sélection optimaux sur la prolificité dans les noyaux "femelles", dans le cas du croisement triple avec le Piétrain, en fonction des conditions d'actualisation et des taux de renouvellement (cas où l'indice de prolificité tient compte de la seule performance de la mère).

Optimum selection rates on litter size in the "female "nucleus herds, in the 3-way cross with Pietrain, according to the discounting parameters and the replacement rates (assuming that selection index on litter size only includes dam performance)

\begin{tabular}{|c|c|c|c|c|c|c|c|c|c|c|}
\hline \multicolumn{2}{|c|}{$\begin{array}{l}\text { Taux d'actualisation (p. Iоo) } \\
\text { (discounting rate) }\end{array}$} & \multicolumn{3}{|c|}{ o } & \multicolumn{3}{|c|}{ IO } & \multicolumn{3}{|c|}{20} \\
\hline $\begin{array}{l}\text { Situation de } \\
\text { renouvellement } \quad(\mathrm{I}) \\
\text { (rule for replacement })\end{array}$ & $\begin{array}{r}\text { Durée d'actualisa- } \\
\text { tion (discoun- } \\
\text { ting period) }\end{array}$ & Io & 20 & 30 & Io & 20 & 30 & Io & 20 & 30 \\
\hline $\operatorname{III} a$ & 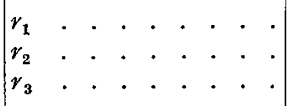 & $\begin{array}{l}88 \\
98 \\
67\end{array}$ & $\begin{array}{l}94 \\
85 \\
56\end{array}$ & $\begin{array}{l}90 \\
7 \mathrm{I} \\
45\end{array}$ & $\begin{array}{l}86 \\
97 \\
67\end{array}$ & $\begin{array}{l}94 \\
86 \\
56\end{array}$ & $\begin{array}{l}90 \\
75 \\
49\end{array}$ & $\begin{array}{l}84 \\
97 \\
67\end{array}$ & $\begin{array}{l}94 \\
88 \\
59\end{array}$ & $\begin{array}{l}91 \\
79 \\
53\end{array}$ \\
\hline . . . . & 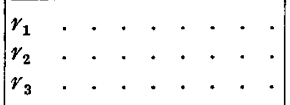 & $\begin{array}{l}90 \\
95 \\
78\end{array}$ & $\begin{array}{l}94 \\
80 \\
67\end{array}$ & $\begin{array}{l}90 \\
65 \\
54\end{array}$ & $\begin{array}{l}88 \\
95 \\
78\end{array}$ & $\begin{array}{l}94 \\
82 \\
69\end{array}$ & $\begin{array}{l}91 \\
69 \\
59\end{array}$ & $\begin{array}{l}87 \\
96 \\
77\end{array}$ & $\begin{array}{l}94 \\
83 \\
70\end{array}$ & $\begin{array}{l}91 \\
73 \\
63\end{array}$ \\
\hline $\mathrm{IIII} c$ & 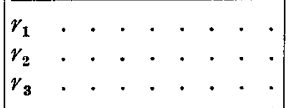 & $\begin{array}{l}7 x \\
92 \\
59\end{array}$ & $\begin{array}{l}78 \\
72 \\
48\end{array}$ & $\begin{array}{l}7 \mathrm{I} \\
57 \\
37\end{array}$ & $\begin{array}{l}70 \\
91 \\
59\end{array}$ & $\begin{array}{l}78 \\
74 \\
49\end{array}$ & $\begin{array}{l}7^{2} \\
6 \mathrm{r} \\
4 \mathrm{r}\end{array}$ & $\begin{array}{l}68 \\
91 \\
59\end{array}$ & $\begin{array}{l}78 \\
77 \\
51\end{array}$ & $\begin{array}{l}73 \\
65 \\
44\end{array}$ \\
\hline $\operatorname{III} d$ & $\begin{array}{lllllllll}r_{1} & \cdot & \cdot & \cdot & \cdot & \cdot & \cdot & \cdot \\
r_{2} & \cdot & \cdot & \cdot & \cdot & \cdot & \cdot & \cdot \\
r_{3} & \cdot & \cdot & \cdot & \cdot & \cdot & \cdot & \cdot \\
\end{array}$ & $\begin{array}{l}72 \\
87 \\
68\end{array}$ & $\begin{array}{l}79 \\
66 \\
56\end{array}$ & $\begin{array}{l}7 \mathrm{I} \\
5^{\circ} \\
44\end{array}$ & $\begin{array}{l}70 \\
87 \\
68\end{array}$ & $\begin{array}{l}79 \\
68 \\
58\end{array}$ & $\begin{array}{l}73 \\
53 \\
49\end{array}$ & $\begin{array}{l}68 \\
87 \\
68\end{array}$ & $\begin{array}{l}79 \\
70 \\
60\end{array}$ & $\begin{array}{l}74 \\
58 \\
53\end{array}$ \\
\hline
\end{tabular}

(1) Voir tableau I (See table I).

(2) En p. roo (in p. 10o).

L'effet des conditions d'actualisation sur les valeurs optimales des taux de sélection sur la prolificité dans les noyaux "femelles » s'explique par l'augmentation avec le temps de l'importance relative, dans le bénéfice $\mathbf{B}_{s}$, de ce que nous avons appelé 1 'effet conjoint des deux types de sélection, c'est-à-dire le terme $\Delta \mathrm{G}_{y} \delta \Delta \mathrm{G}^{\prime} x$ de la relation (Io). Du fait de la sélection sur la performance de production, la marge moyenne retirée de la vente des produits terminaux augmente avec le temps. La sélection sur la prolificité est d'autant plus justifiée que cette marge est élevée et on comprend dès lors que l'intérêt de cette sélection augmente avec la durée d'actualisation. 


\section{b) Effet sur le gain dî̀ à la sélection:}

L'effet du taux d'actualisation sur le gain dû à la sélection (en appliquant les taux optimaux de sélection sur la prolificité) est pratiquement le même, quels que soient les taux de renouvellement des reproducteurs des noyaux "femelles ", l'information utilisée pour la sélection sur la prolificité et le plan de croisement (tableau 9). L'actualisation diminue d'autant plus ces bénéfices - par rapport à leur valeur non actualisée - que la durée est longue, ce qui traduit l'augmentation dans le temps des recettes instantanées (perçues au cours d'un semestre). Contrairement aux recettes dues au croisement, les recettes dues à la sélection augmentent de façon plus que linéaire avec le temps. Ces recettes ont en effet trois origines comme l'indique la relation (ro) : la sélection sur la performance de production $\left(\Delta \mathrm{G}_{\mathbf{y}} \beta\right)$, la sélection sur la prolificité $\left(\gamma \Delta \mathrm{G}_{\mathbf{x}}^{\prime}\right)$ et l'effet conjoint de ces deux sélections $\left(\Delta \mathrm{G}_{\mathbf{y}} \delta \Delta \mathrm{G}_{\mathbf{x}}{ }_{\mathbf{x}}\right)$. Comme les taux de sélection optimaux sur la prolificité diminuent quand la durée d'actualisation augmente, la part des deux dernières origines croît avec ce facteur.

Par ailleurs, alors que dans le croisement en retour les deux noyaux de race pure sont sélectionnés de façon identique, dans le croisement triple les truies et verrats du noyau de sélection de la souche mâle ne sont pas choisis sur la prolificité de leur mère. On a donc représenté sur les figures $3^{\mathrm{A}}$ et $3^{\mathrm{B}}$ la part des recettes dues à la sélection sur la prolificité et à l'effet conjoint des deux sélections, dans les recettes totales d'une part, dans les recettes dues à la sélection des deux noyaux Large White et Landrace Français d'autre part. Dans le cas du croisement en retour, cette part est toujours négligeable ( $\mathrm{p}$. I Ioo au maximum) et seul le cas du

TABLEAU 9

Influence des conditions d'actualisation sur le gain dù à la sélection $\left(B_{s}\right)$

Influence of discounting parameters on the gain due to selection $\left(B_{s}\right)$

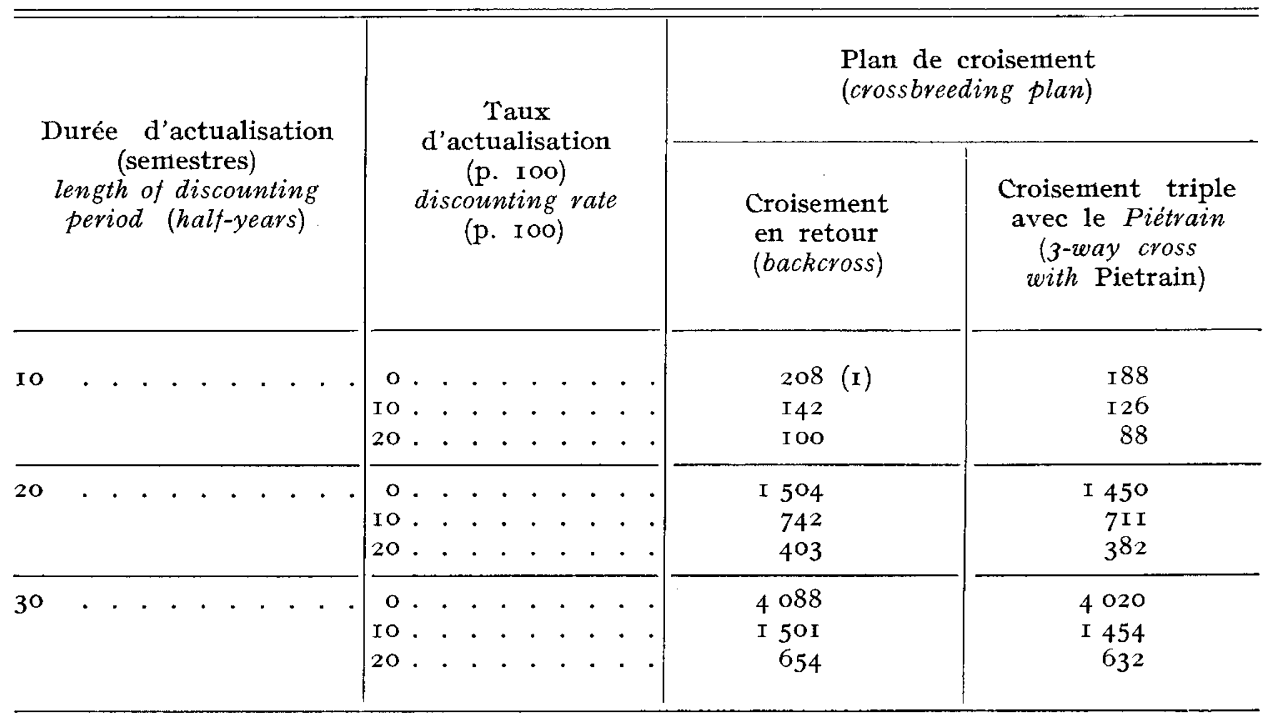

(1) En millions de francs. 


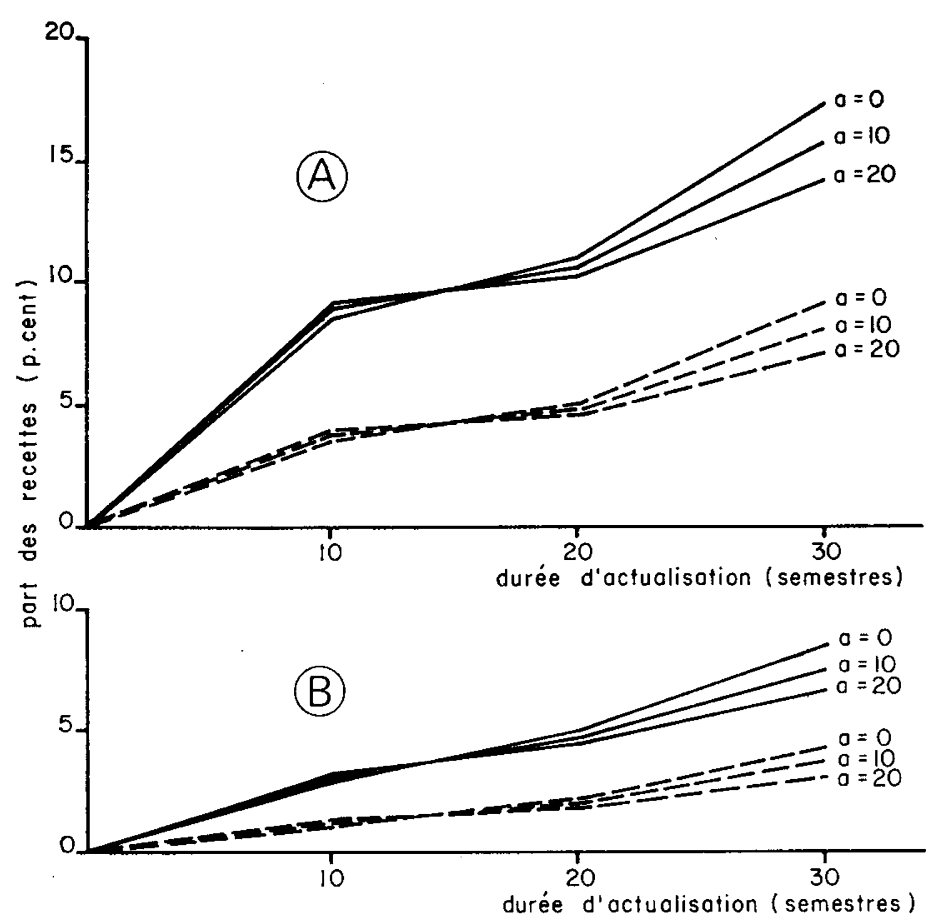

FIG. 3. - Part des recettes due à la sélection sur la prolificité et à l'effet conjoint des deux types de sélection $(A)$ dans les recettes dues à la sélection des deux noyaux "femelles", $(B)$ dans les recettes totales dues à la sélection, en fonction de la durée et du taux d'actualisation (cas du croisement triple avec le Piétrain et de la sélection sur la prolificité de la mère).

Relative importance of the veturns due to the selection on litter size and to the joint effect of the two types of selection $(A)$ in the veturns due to selection in the two "female "nucleus herds, $(B)$ in the total veturns due to selection, as a function of the length of discounting period and discounting rate (case of the 3 -way cross with Pietrain and of selection on dam prolificacy).

trait plein : 2 mises bas/femelle, I période de saillie/mâle

(full line: 2 farrowings/female, I mating period/male)

trait pointillé : I mise bas/femelle, 2 périodes de saillie/mâle

(dotted line: I farrowing/female, 2 mating periods/male)

$a=$ taux d'actualisation (discounting rate).

croisement triple avec le Piétrain a été représenté, en prenant comme exemple les deux situations de renouvellement les plus extrêmes de ce point de vue. Comme prévu, ces parts augmentent avec la durée d'actualisation. L'influence du taux d'actualisation est moins simple puisqu'elle s'inverse dans le temps, les solutions non actualisées correspondant aux parts les plus grandes pour une durée de 30 semestres. Cependant, les variations de ces parts avec le taux d'actualisation restent faibles. Enfin, on notera que l'importance, dans les recettes dues à la sélection, de celles qui proviennent de la sélection sur la prolificité est assez modeste puisqu'elles ne dépassent jamais 20 p. roo du total.

Par la suite, sauf indication contraire, les résultats seront donnés pour une durée d'actualisation de 30 semestres et un taux de Io p. Ioo, ces valeurs nous semblant les plus raisonnables d'un point de vue économique. 


\section{Taux de renouvellement des reproducteurs dans les noyaux "femelles"}

Les premiers résultats obtenus dans le cas du croisement en retour et du croisement triple avec un mâle Piétrain ont montré qu'il est toujours préférable de renouveler les mâles aussi rapidement que possible. Pour les deux autres croisements triples (avec la souche Large White "viande" ou le Landrace Belge), nous nous sommes donc limités au cas d'une seule période de saillie par mâle dans les noyaux "femelles".

\section{a) Effet sur les taux de sélection:}

Les résultats concernant le croisement triple avec un mâle Piétrain figurent au tableau 8. Rappelons que dans le cas du croisement en retour, la sélection sur la prolificité ne se justifie pratiquement jamais, les taux de sélection ne différant notablement de roo p. Ioo que dans le cas des durées d'actualisation longues et dans le cas d'un renouvellement lent des femelles. Les taux de sélection optimaux trouvés pour les deux autres croisements triples obéissent aux mêmes tendances que ceux présentés au tableau 8 et seront discutés ultérieurement.

En ce qui concerne les mâles et les femelles destinés au renouvellement des noyaux de race pure, la sélection sur la prolificité se justifie d'autant plus que les taux de renouvellement sont faibles : en effet, d'une part les possibilités de sélection sont plus grandes ( $\lambda_{1}$ et $\lambda_{2}$ sont plus petits), d'autre part l'information sur ce caractère est plus précise quand les truies sont connues sur deux mises bas. L'effet du taux de renouvellement des femelles sur la valeur optimale des taux de sélection est d'ailleurs plus important que celui des mâles.

Le choix des mâles pour le renouvellement des étages inférieurs est moins simple à interpréter puisque le taux optimal de sélection sur la prolificité est minimal dans le cas de deux mises bas par femelle et d'une période de saillie par mâle, maximal dans le cas d'une mise bas par femelle et deux périodes de saillie par mâle et intermédiaire pour les taux de renouvellement simultanément petits ou grands.

\section{b) Effet sur le gain dî̀ à la sélection}

Comme on le voit à la figure 4 , on a intérêt à renouveler les mâles aussi vite que possible dans les deux plans de croisement qui ont été étudiés de ce point de vue; la réduction du gain dû à la sélection quand on passe d'une à deux périodes de saillie par mâle est plus marquée dans le cas du croisement en retour (environ 5 p. roo) que dans le cas du croisement triple avec le Piétrain ( 2 à 2,5 p. IOo). Le taux optimum de renouvellement des femelles varie avec le plan de croisement : il correspond à une mise bas par truie pour le croisement en retour et à deux mises bas par truie pour les croisements triples. Remarquons que pour le croisement en retour, le fait de passer à deux mises bas par truie entraîne une réduction du gain dû à la sélection de I,3 p. Ioo seulement. Pour les croisements triples, le passage de deux à une mise bas par truie entraîne une réduction de ce gain qui atteint près de $3 \mathrm{p}$. roo quand la sélection sur la prolificité porte sur l'indice le plus précis (mère + autres apparentées). Ces résultats sur les taux optimaux de renouvellement sont valables quelles que soient les valeurs des autres facteurs de variation.

\section{Type d'information sur la prolificité}

Rappelons qu'il a été envisagé quatre types d'information : prolificité de la mère seule d'après sa première portée ou d'après ses deux premières portées, prolificité de la mère et d'autres apparentées d'après leur première portée ou d'après 


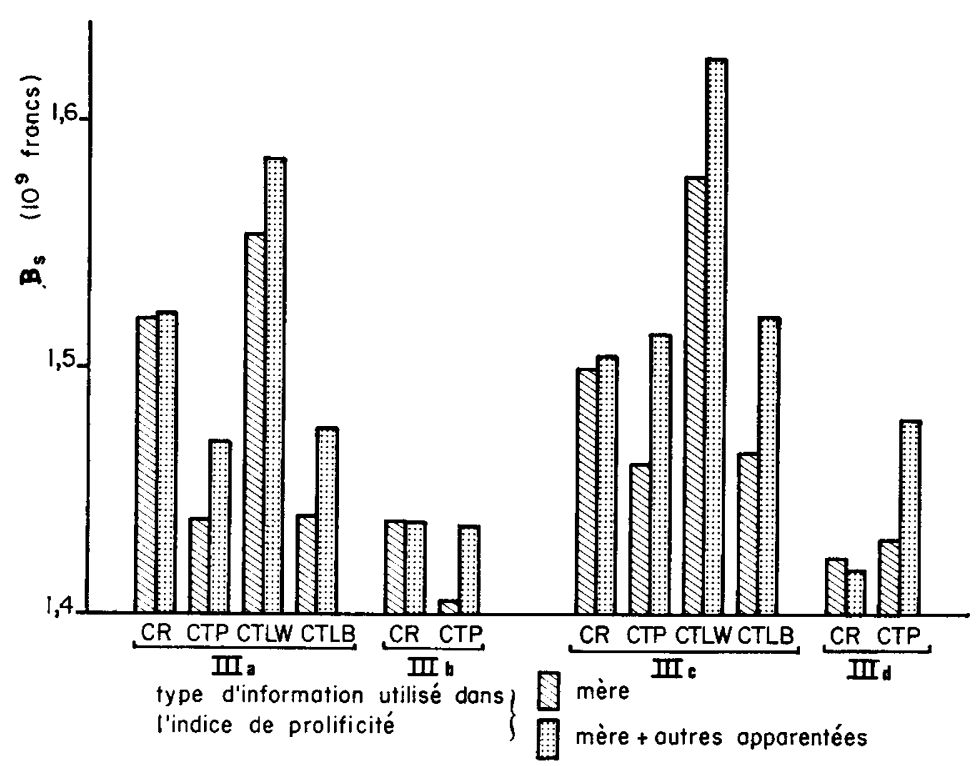

Fig. 4. - Influence des taux de venouvellement dans les noyaux "femelles ", du type d'information utilisé dans l'indice de prolificité et du plan de croisement sur le gain dù à la sélection $\left(B_{8}\right)$.

Influence of the replacement rates in the "female "nucleus herds, the type of information used in the selection index on prolificacy and the crossbreeding plan on the gain due to selection $\left(B_{s}\right)$.

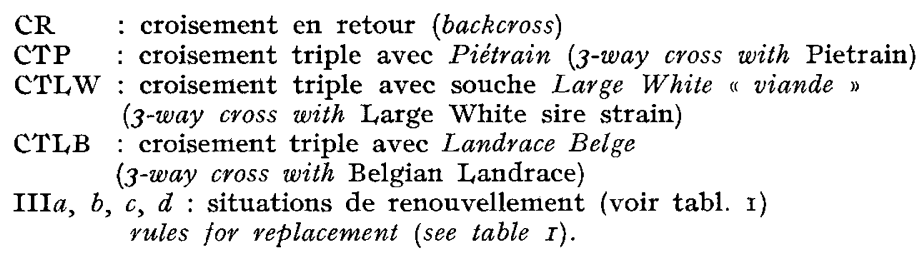

leurs deux premières portées. L'influence du nombre de mises bas par femelle a déjà été étudiée puisque ce nombre est en relation directe avec le taux de renouvellement.

L'utilisation pour la sélection d'une information accrue sur la prolificité des femelles apparentées aux candidats permet une augmentation du poids accordé à la prolificité dans le choix des reproducteurs et doit donc se traduire par une diminution des taux de sélection sur ce caractère et une augmentation du gain dû à la sélection.

\section{a) Effet sur les taux de sélection:}

On trouvera sur la figure 5 les taux de sélection optimaux à pratiquer sur l'indice de prolificité, pour chacun des plans de croisement. Les conclusions sont identiques pour des conditions d'actualisation ou des taux de renouvellement différents.

En ce qui concerne le croisement en retour, la prise en compte d'une information plus précise sur la prolificité ne modifie pratiquement pas les taux de sélection optimaux sur ce caractère. Pour les croisements triples, ces taux diminuent de Io à 20 points. 


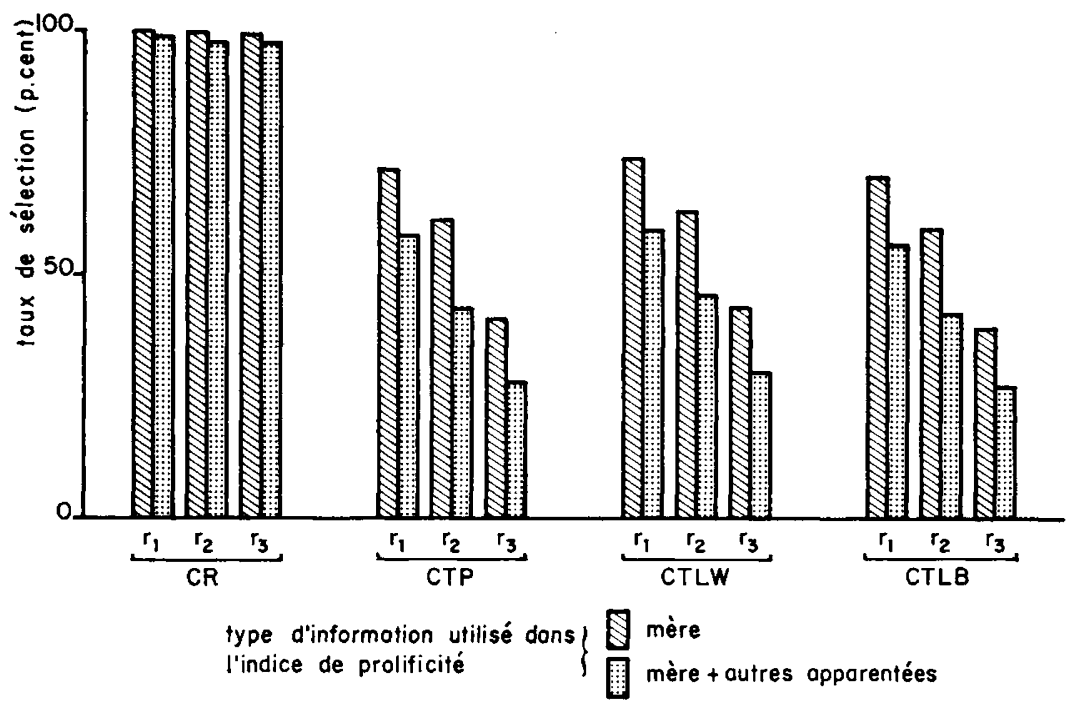

FIG. 5. - Taux de sélection optimaux sur la prolificité dans les noyaux "femelles ", en fonction du plan de croisement et du type d'information utilisé dans l'indice de prolificité (ces valeurs correspondent à l'optimum des taux de renouvellement des reproducteurs).

Optimum selection rates on litter size in the "female " nucleus herds, according to the crossbreeding plan and the type of information used in the selection index on prolificacy (these values correspond to the optimum replacement rates of breeding animals).

$r_{1}=$ taux de sélection des femelles pour le renouvellement des noyaux

(selection rate of females retained for replacement of nucleus herds)

$r_{2}=$ taux de sélection des mâles pour le renouvellement des noyaux

(selection rate of males retained for replacement of nucleus herds)

$r_{3}=$ taux de sélection des mâles pour le renouvellement des étages inférieurs

(selection rate of males moved to lower tiers)

CR, CTP, CTLW, CTLB : voir fig. 4 (see fig. 4).

b) Effet sur le gain dî̀ à la sélection:

Comme prévu, dans le cas des croisements triples, la part des recettes due à la sélection sur la prolificité et à l'effet conjoint de la sélection sur ce caractère et de la sélection sur la performance de production est plus importante si les performances de toutes les femelles apparentées sont prises en compte dans la sélection sur la prolificité (tableau ro). Cette part est environ doublée et le gain dû à la sélection est supérieur de 3 à $4 \mathrm{p}$. Ioo à celui qui avait été trouvé précédemment.

En ce qui concerne le croisement en retour pour lequel les taux de sélection sur la prolificité ne sont pratiquement pas modifiés, le gain dû à la sélection est inchangé.

\section{Plans de croisement}

a) Effet sur les taux de sélection (fig. 5):

Comme nous l'avons vu à plusieurs reprises, la sélection sur la prolificité ne se justifie pas dans le cas du croisement en retour. En ce qui concerne le croisement triple, les valeurs optimales des taux de sélection sur la prolificité varient avec la race du mâle terminal mais les différences restent minimes. 
Influence de la précision de l'indice de sélection sur la prolificité sur l'importance relative (en p. Ioo) de la part due à la sélection sur la prolificité et à l'effet conjoint des deux types de sélection (I) dans le gain total dù à la sélection (2) dans le gain dù à la sélection des noyaux "femelles": cas des croisements triples.

Influence of the precision of the selection index on litter size on the relative importance (in $p$. Ioo) of the part due to selection on litter size and to joint effect of the two selections (I) in the total gain due to selection (2) in the gain due to selection of the "female "nucleus herds: case of 3-way crosses.

\begin{tabular}{|c|c|c|c|c|c|}
\hline \multirow{2}{*}{\multicolumn{2}{|c|}{$\begin{array}{c}\text { Information utilisée } \\
\text { dans l'indice de prolificité } \\
\text { (information used in the prolificacy index) }\end{array}$}} & \multicolumn{2}{|c|}{$\begin{array}{l}\text { Mère } \\
(\text { dam })\end{array}$} & \multicolumn{2}{|c|}{$\begin{array}{c}\text { Mère }+ \text { tantes } \\
\text { et demi-tantes } \\
(\text { dam }+ \text { aunts } \\
\text { and half-aunt })\end{array}$} \\
\hline & & (I) & (2) & (I) & (2) \\
\hline \multirow{3}{*}{$\begin{array}{l}\text { Crcisement } \\
\text { triple } \\
\text { avec } \\
\text { (3-way cross with) }\end{array}$} & Large White "viande". & 6,7 & $\mathrm{I} 4,3$ & I 2,7 & $26, \mathrm{I}$ \\
\hline & Piétrain . . . . . & 7,5 & I 5,7 & I3,9 & 27,9 \\
\hline & $\begin{array}{l}\text { Landrace Belge } \\
\text { (Belgian Landrace) }\end{array}$ & $8, \mathbf{I}$ & I6,7 & I 4,7 & 29,4 \\
\hline
\end{tabular}

b) Effet sur le gain dî̀ à la sélection:

Conformément aux résultats obtenus pour les taux de sélection, la part du gain due à la sélection sur la prolificité est un peu plus élevée pour le croisement triple avec le Landrace Belge que pour le croisement triple avec le Piétrain ou le Large White "viande" (tabl. Io). Cependant, sur le plan des gains totaux dus à la sélection, l'utilisation d'une souche Large White "viande " comme lignée mâle terminale est supérieure aux autres plans de croisement (fig. 4). En outre, en supposant que les taux de renouvellement optimaux dans les noyaux "femelles" sont pratiqués pour chaque plan de croisement, le croisement en retour, vis-à-vis du gain dû̀ à la sélection, présente un léger avantage sur les croisements triples avec le Piétrain ou le Landrace Belge quand l'indice de sélection sur la prolificité inclut toute l'information sur les apparentées mais cet avantage est plus substantiel quand l'indice de prolificité n'inclut que les performances de la mère. Il apparaît en définitive que l'effet de la lignée mâle terminale sur la taille de portée a une influence déterminante sur les différences de rentabilité de la sélection.

\section{C. - Résultats globaux}

Un schéma d'amélioration génétique est défini ici par le plan de croisement et le plan de sélection mis en œuvre. Ce dernier est caractérisé par les taux de renouvellement et de sélection pratiqués. De façon générale, on peut se poser deux questions : 
I. - Quel est le schéma le plus intéressant, c'est-à-dire apportant le bénéfice maximum?

2. - Quel est l'intérêt pratique de la sélection sur la prolificité dans ce schéma?

\section{Détermination du schéma d'amélioration génétique optimum}

Le bénéfice total (B) résultant de l'application des différents schémas d'amélioration génétique étudiés est la somme du gain dû à la sélection $\left(\mathrm{B}_{8}\right)$ et du gain dû au croisement $\left(B_{c}\right)$. Ce dernier n'étant pas affecté par les taux de sélection sur la prolificité et étant très peu affecté par les taux de renouvellement dans les noyaux "femelles ", au moins pour la marge de variation envisagée pour ceux-ci, les valeurs de ces taux qui maximisent $B$ sont celles qui maximisent $B_{s}$ : elles ont été données au paragraphe $B .2 . b$ et à la figure 5 , pour une durée d'actualisation de 30 semestres et un taux d'actualisation de ro p. Ioo.

Les bénéfices $\mathrm{B}, \mathrm{B}_{c}$ et $\mathrm{B}_{s}$ qui correspondent à ces valeurs optimales pour les quatre plans de croisement sont représentés sur la figure 6 .

Le croisement triple avec le Piétrain est inférieur au croisement en retour aussi bien pour le gain dû̀ à la sélection que pour le gain dû au croisement, et donc pour le bénéfice total. Le croisement triple avec la souche Large White "viande " est supérieur au croisement en retour pour les deux catégories de gains et se révèle le plus intéressant des quatre plans de croisement. Le croisement triple avec le Landrace Belge est le plus efficace en ce qui concerne le gain dû au croisement mais il ne permet pas d'espérer un gain dû à la sélection aussi élevé que le croisement en retour : toutefois, il est globalement supérieur à ce dernier.

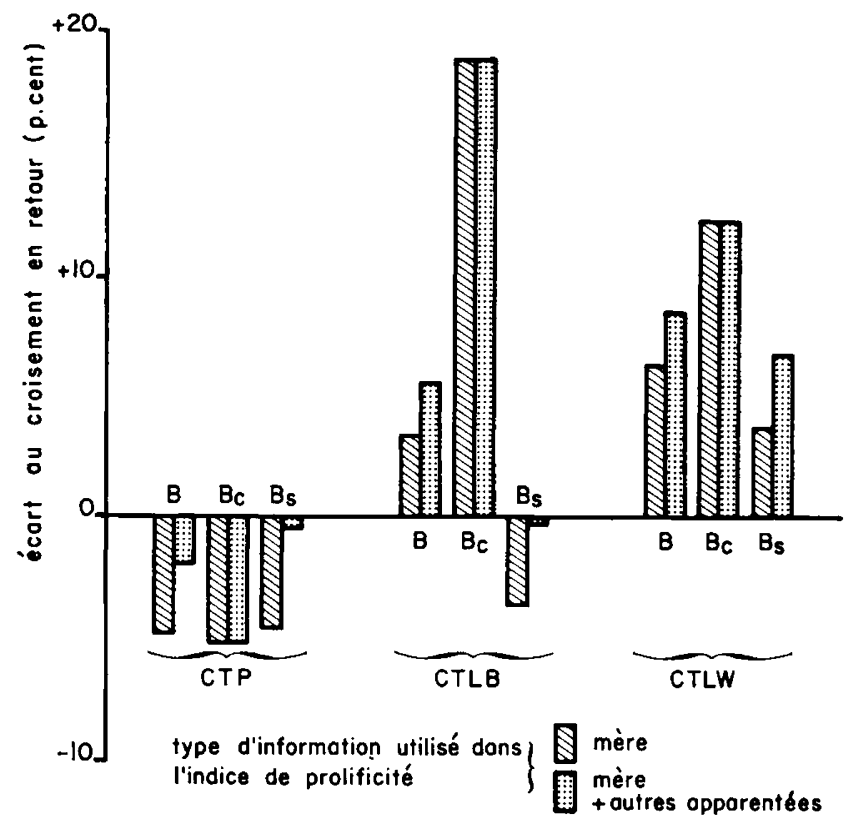

FIc. 6. - Bénéfice total $(B)$, gain dû au croisement $\left(B_{c}\right)$ et gain dù à la sélection $\left(B_{8}\right)$ pour chacun des plans d'amélioration génétique (exprimés en écart au croisement en retour).

Total benefit $(B)$ gain due to crossing $\left(B_{8}\right)$ and gain due to selection $\left(B_{8}\right)$ in each plan of genetic improvement (in deviation from the backcross).

CTP, CTLB, CTLW : voir fig. 4 (see fig. 4). 
Apport de la sélection sur la prolificité dans le bénéfice total $(B)$ et dans le gain dù à la sélection $\left(B_{8}\right)$ dans le cas des croisements triples ( $\mathbf{I}$ )

Increase of the total benefit $(B)$ and the gain due to selection $\left(B_{8}\right)$ as a result of selection on prolificacy in the case of 3-way crosses

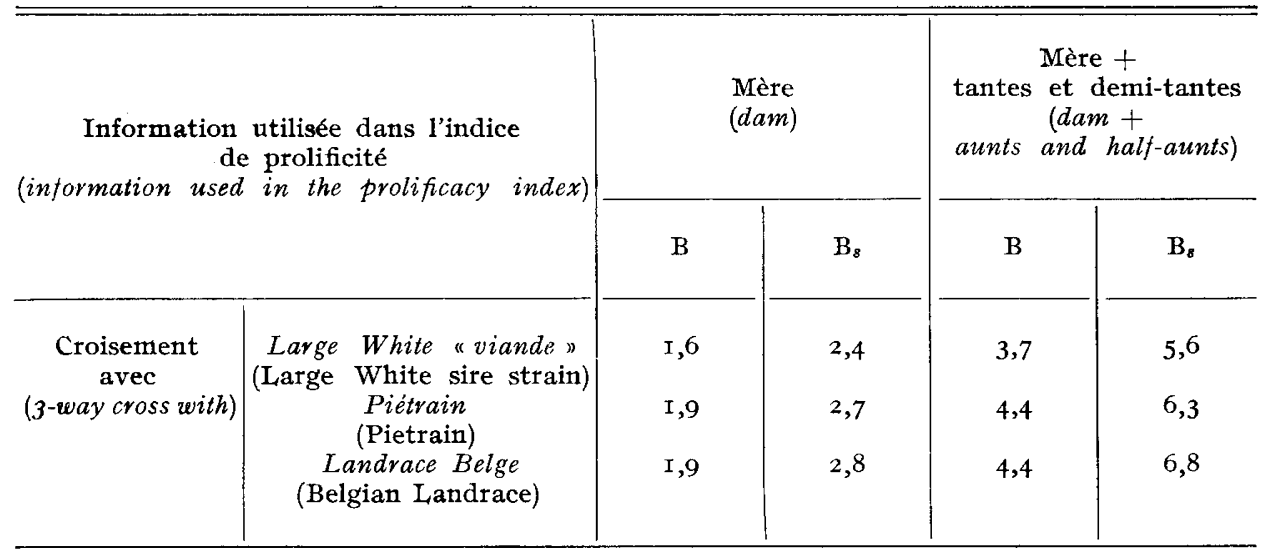

(1) En pourcentage des valeurs de $B$ et $B_{8}$ correspondant à la situation où la prolificité n'est pas prise en compte dans la sélection. (In p. cent of the values of $\mathbf{B}$ and $\mathbf{B}_{8}$ corresponding to the case where no selection is made on litter size).

2. Importance de la sélection sur la prolificité dans la situation optimale pour chaque plan de croisement

Nous avons vu que les taux optimaux de sélection sur l'indice de prolificité sont très différents de roo $\mathrm{p}$. Ioo dans le cas des croisements triples et que la part due à la sélection sur ce caractère et à l'effet conjoint des deux types de sélection dans les gains totaux dus à la sélection varie entre 6 et I 5 p. Ioo selon le plan de croisement et le type d'information utilisé dans l'indice de prolificité. Cependant, l'effort de sélection total, caractérisé par les paramètres $\lambda_{1}, \lambda_{2}$ et $\lambda_{3}$, est fixé et la valeur moyenne des reproducteurs pour les caractères de croissance et d'adiposité est inférieure avec ce choix optimal à celle qui serait obtenue si la sélection portait seulement sur ces caractères. On trouvera, au tableau II, le surcroît de gains dus à la sélection d'une part, le surcroît de bénéfice total d'autre part, que la sélecsion préalable sur la prolificité permet d'espérer, par rapport à la situation d'une sélection sur la seule performance de production.

L'introduction de la sélection sur la prolificité apporte relativement peu à la rentabilité des schémas : 1'augmentation du bénéfice total et des gains dus à la sélection ne dépasse pas respectivement 5 et $7 \mathrm{p}$. Ioo. Toutefois, il est bon de remarquer qu'en valeur relative, ce supplément est du même ordre de grandeur que les différences trouvées entre plans de croisement pour les deux mêmes critères (fig. 6). En particulier, quand on applique les valeurs optimales des taux de renouvellement et de sélection, 1'avantage du croisement triple avec un mâle Large White "viande " sur le croisement en retour n'est que de 8,6 p. Ioo pour le bénéfice total. 


\section{IV. - Discussion et conclusions}

Les conclusions auxquelles nous sommes arrivés sont, comme dans toute étude prévisionnelle de ce genre, étroitement liées à la fois aux hypothèses de base du modèle choisi et aux valeurs données aux paramètres fixés de ce modèle. L'ampleur même du problème posé et la nécessité, pour des raisons de volume des calculs, de limiter le nombre de variables nous ont imposé de faire de nombreux choix a priori : aussi importe-t-il de bien garder à l'esprit les hypothèses du modèle et les valeurs des paramètres fixés pour apprécier la signification réelle de nos résultats. Certaines hypothèses méritent d'être brièvement discutées avant de commenter les résultats et de tirer les principales conclusions de cette étude.

\section{I. - Remarques sur le modèle.}

L'un des problèmes soulevés par les études économiques sur les schémas d'amélioration génétique des espèces domestiques est le choix des conditions d'actualisation et en particulier du taux d'actualisation. Ce dernier point a été discuté récemment par SMITH (I978) qui estime trop élevés les taux communément retenus ( 8 à $\mathrm{I} 5 \mathrm{p}$. roo) dans les études génético-économiques, dans la mesure où ils conduisent à sous-estimer la valeur des recettes résultant des gains génétiques espérés. Il juge préférable d'en soustraire le taux d'in flation et, sur cette base, propose d'utiliser un taux d'actualisation de $3 \mathrm{p}$. Ioo dans ce type d'étude. Si l'on adopte cette ligne de conduite, il y a lieu de considérer comme les plus intéressants les résultats obtenus ici pour les valeurs o et ro p. roo du taux d'actualisation, qui encadrent la valeur recommandée par SMrTH. On peut estimer cependant qu'indépendamment de l'aspect strictement monétaire, le choix d'un taux d'actualisation relativement élevé (Io p. Ioo par exemple) est une façon de prendre en compte le facteur " risque " inhérent à tout investissement et que cette attitude semble particulièrement souhaitable si l'on se place du point de vue d'une entreprise privée comme nous l'avons fait ici d'une certaine manière. De toute façon le choix final de tel ou tel taux d'actualisation revient au " décideur " et dans une étude prévisionnelle comme celle-ci, il est préférable de donner les résultats pour une gamme de taux d'actualisation.

Par ailleurs, il convient d'attirer l'attention sur le caractère un peu artificiel du cadre économique de cette étude : il s'agit d'une organisation économique complètement " intégrée ", où toutes les décisions sont prises par un maître-d'œuvre unique, dont le volume de production est suffisamment petit vis-à-vis de la production nationale pour que ces décisions n'aient pas de répercussion sur les conditions du marché, en particulier les prix, et dont 1'objectif est de maximiser le bénéfice qu'elle retire de la production de viande de porc, le nombre de mères de l'étage terminal étant supposé fixé. Sur ce dernier point, d'autres hypothèses auraient pu être faites : par exemple, dans la ligne des études de MoAv (I966) et de DickERson (I973), nous aurions pu supposer la constance du nombre de porcs vendus. Quant à l'hypothèse d'intégration, elle soulève le problème important des relations entre les partenaires d'un tel schéma : la difficulté de ce problème est évoquée par BICHARD et WILSON (I974).

En ce qui concerne le modèle démographique, nous avons en particulier supposé que la population des femelles était en état d'équilibre à l'instant initial, le plan de croisement en retour étant appliqué depuis un temps assez long pour que 
les effectifs des cohortes de femelles ne varient plus d'une période à la suivante. De la même façon, dans le cas des croisements triples, nous avons fait l'hypothèse que le passage à 1'utilisation exclusive de verrats de la lignée mâle spécialisée dans les élevages de production était réalisé le plus rapidement possible. Le problème de la mise en place d'un plan de croisement dans une population ne peut être étudié de façon complète à 1'aide du type de modèle que nous avons utilisé : la programmation linéaire pourrait être un bon outil pour une telle analyse (CHApPuis et SoURIE, I974). Il est probable en particulier qu'au cours de cette phase de mise en place, le plan de sélection optimal à appliquer dans les noyaux "femelles " soit différent de celui que nous proposons.

Nous avons supposé qu'il y avait, parmi les truies $F_{1}$ des élevages commerciaux, autant de filles de mâles Large White que de filles de mâles Landrace Français et que dans le croisement en retour, ces femelles étaient accouplées à parts égales à des mâles de ces deux races. Cette situation ne correspond pas à l'optimum du gain dû au croisement. D'une part, un avantage des filles de verrats Landrace et de truies Large White sur les femelles issues du croisement réciproque a été trouvé pour la taille de portée au sevrage : cet avantage est de 0,07 porcelet dans l'étude de SmiTh et KING (I964) et de o,2 I porcelet dans l'étude de L,EGAULiT et al. (I975). D'autre part, dans le croisement en retour, l'utilisation exclusive de verrats Large White dans l'étage terminal conduirait à des recettes dues au croisement plus importantes puisque le mâle Large White présente un avantage, vis-àvis du verrat Landrace Français, à la fois pour la marge unitaire et pour l'effet sur la taille de portée. Il est donc évident que sur le plan du gain dû au croisement, la situation retenue ici pour l'option croisement en retour (égale répartition entre les quatre types génétiques possibles) est inférieure à la situation où tous les produits terminaux proviennent du croisement entre verrats Large White et truies $\mathrm{F}_{1}$ filles de verrats Landrace Français; le croisement en retour s'en trouve très probablement défavorisé par rapport aux croisements triples mais 1'égale répartition des types génétiques nous a paru être la situation de référence la plus appropriée à l'heure actuelle. La situation optimale de ce point de vue serait une situation de compromis tenant compte des coûts d'entretien des noyaux de race pure et variant avec le niveau génétique des reproducteurs, donc d'un semestre à l'autre.

Nous avons fait l'hypothèse que les paramètres génétiques sont constants dans le temps, qu'il s'agisse des variances et covariances génétiques ou des effets d'hétérosis. La corrélation génétique entre taille de portée et performance de production a été supposée nulle : cette hypothèse est étayée, nous l'avons vu, par la plupart des résultats expérimentaux disponibles mais il est probable, au vu des conclusions de Smith (I964), que l'existence d'une corrélation génétique défavorable aurait des conséquences importantes sur les réponses au problème que nous avons abordé. Pour évaluer l'effet du croisement, nous avons supposé que l'effet d'hétérosis, exprimé en unité physique du caractère, était invariant dans le temps, donc indépendant de la moyenne des races parentales, laquelle évolue du fait de la sélection. Cette hypothèse, qui a l'avantage de conduire à des calculs plus simples, peut être discutée : le problème d'une variation éventuelle de l'effet d'hétérosis pour un caractère dont la moyenne est modifiée par la sélection des populations parentales (en supposant évidemment qu'il n'y a d'accroissement du coefficient de consanguinité moyen de celles-ci) ne semble pas de toute façon avoir reçu de réponse sur le plan expérimental chez le Porc, comme le remarque SELLIER (I976b).

En ce qui concerne les hypothèses pouvant avoir une influence sur l'efficacité globale de la sélection et donc sur le bénéfice total, plusieurs points sont à souligner : 
- l'hypothèse d'ajustement des effectifs des noyaux de sélection aux besoins de renouvellement des étages inférieurs pourrait être levée : une taille plus grande des noyaux de race pure et donc un nombre plus grand de candidats à la sélection permettraient d'augmenter les pressions de sélection et d'accroître la valeur génétique des reproducteurs destinés aux étages de multiplication et de production. Il faudrait alors déterminer la taille optimum des noyaux de sélection, compte tenu des coûts de production et de contrôle des candidats supplémentaires et de l'accroissement de supériorité génétique des animaux retenus. On peut rappeler les conclusions de BICHARD et WILson (I974) au sujet de la politique de renouvellement des femelles dans une structure de multiplication identique à celle considérée ici : comparant le bénéfice par porc et le coût de sélection et de multiplication (" selection and multiplication overhead "), ils ont montré que la meilleure solution consiste à transférer toutes les jeunes truies disponibles des noyaux de sélection aux élevages de multiplication d'une part, des élevages de multiplication aux élevages de production d'autre part. Cette conclusion rejoint 1 'hypothèse que nous avons retenue;

- nous avons supposé négligeable 1'accroissement des pressions de sélection résultant du progrès génétique pour la taille de portée et donc de l'augmentation du nombre de candidats à la sélection. Cette hypothèse, raisonnable avec les durées d'actualisation envisagées, conduit à sous-estimer à très long terme l'intérêt de la sélection sur la prolificité et sans doute aussi à défavoriser les plans de croisement dans lesquels, d'après nos résultats, cette sélection se justifie. Dans le même ordre d'idées, on peut noter que les taux de sélection trouvés sont des taux moyens estimés pour l'ensemble de la durée d'actualisation et qu'une analyse plus fine devrait fournir les taux de sélection optimaux pour chaque période. Ce nouveau problème comprendrait non plus 6 mais $6 T$ variables, $T$ étant la durée d'actualisation. En pratique, pour des raisons de coût de calcul, la méthođe d'optimisation que nous avons utilisée ne pouvait permettre la résolution d'un problème de cette taille;

- LEgault (I970a) a montré que, dans le cas d'une sélection en deux étapes sur la prolificité de la mère avec un taux $r$, puis sur la performance de production avec un taux $p-$, les possibilités totales de sélection dépendent de la taille moyenne des familles retenues après le premier stade, cette taille augmentant avec la pression de sélection sur la prolificité de la mère. En toute rigueur le produit $r p$ des taux de sélection n'est donc pas égal au paramètre $\lambda$ tel qu'il a été défini ici mais à $\lambda \mu /(\mu+\iota \sigma)$ où $\mu$ et $\sigma$ sont la moyenne et l'écart-type phénotypique de la taille de portée et ‘ l'intensité de sélection sur ce caractère. Si la simplification que nous avons adoptée est sans effet dans le cas du croisement en retour où pratiquement aucune sélection sur la prolificité n'est à envisager $(\iota=0)$, elle défavorise légèrement les croisements triples pour lesquels le gain dû à la sélection a été sous-estimé, sans que l'on puisse préciser a priori comment cette possibilité supplémentaire de sélection se répartit à l'optimum entre les deux groupes de caractères.

\section{2. - Conclusions générales.}

Les résultats concernant l'influence de la durée et du taux d'actualisation sont, dans l'ensemble, conformes à ce qu'on pouvait prévoir et appellent peu de commentaires. Les deux conclusions les plus nettes sont les suivantes: 
- le fait de faire varier les conditions d'actualisation n'affecte pas de façon sensible le mérite relatif des plans de croisement;

- dans le cas des croisements triples, l'intensité optimale de sélection sur la prolificité dans les noyaux "femelles » est d'autant plus forte que la durée d'actualisation est plus longue et le taux d'actualisation plus faible ou, en d'autres termes, que l'on donne plus de "poids " au long terme.

Dans le même ordre d'idées et toujours dans le cas des croisements triples, le fait d'accroître la précision de l'estimation des valeurs génétiques pour la prolificité - en utilisant dans l'indice de sélection non seulement l'information relative à la mère des candidats mais aussi celle relative à d'autres apparentées - a pour conséquence de justifier une pression de sélection plus forte sur ce caractère dans les noyaux "femelles ": ce résultat était attendu dans la mesure où cet accroissement de précision de l'indice de prolificité est supposé n'entraîner aucun coût de sélection supplémentaire. L'utilisation de cet indice de sélection plus précis, dans le cas des croisements triples, a pour autres conséquences de doubler environ, dans le gain dû à la sélection, la part des recettes dues à la sélection sur la prolificité et à ce que nous avons appelé 1'effet conjoint des deux types de sélection, et aussi d'augmenter de 3 à $4 \mathrm{p}$. Ioo le gain total dû̀ à la sélection. Par contre, dans le cas du croisement en retour, l'accroissement de la précision de l'indice n'apporte aucun changement quant à l'intérêt de la sélection sur la prolificité dans les deux noyaux de race pure.

Les taux de renouvellement des reproducteurs des deux sexes dans les noyaux "femelles" sont pratiquement sans influence sur le gain dî au croisement et sur la valeur comparée des plans de croisement. Par contre - et bien que nous n'ayons envisagé ici qu'une amplitude de variation restreinte pour ces taux, visant à "encadrer au plus près " les valeurs optimales trouvées dans diverses situations par OLLIVIER (I974) - ils ont une influence notable sur le gain dû à la sélection. L'effet le plus net concerne le taux de renouvellement des mâles dans le cas du croisement en retour : la différence entre les deux variantes étudiées pour ce taux atteint 5 p. Ioo.

De façon plus générale, la meilleure politique de sélection dans les noyaux "femelles " (taux de sélection et de renouvellement) dépend du plan de croisement pratiqué. Il s'agit là, à notre sens, de l'une des conclusions intéressantes de cette étude et les résultats sur ce point peuvent être résumés ainsi :

- dans le cas du croisement en retour, la sélection sur la prolificité dans les noyaux de race pure ne se justifie pratiquement jamais, même dans la situation a priori la plus "favorable" (conditions d'actualisation privilégiant le long terme et précision accrue de 1'indice de sélection sur la prolificité). Par ailleurs, il y a intérêt à renouveler le plus vite possible les reproducteurs des deux sexes, c'est-à-dire à réformer les mâles après une période de saillie et les femelles après leur première portée. Nous retrouvons là un résultat proche de celui obtenu par OLLIVIER (I974) dans la situation correspondante (sélection individuelle sur la performance de production);

- dans le cas des trois croisements triples étudiés, la sélection sur la prolificité se justifie dans les noyaux "femelles": sur une durée de $\mathrm{I}_{5}$ ans et avec un taux d'actualisation de ro p. Ioo, l'application des taux de sélection optimaux sur l'indice de prolificité incluant l'information relative à la mère et à d'autres apparentées du candidat permet d'accroître d'environ 6 p. Ioo le gain dû à la sélection, par rapport à la situation où la prolificité est ignorée dans la sélection. Ces taux de sélection optimaux sont de l'ordre de 50 à $60 \mathrm{p}$. Ioo pour le renouvellement des femelles des noyaux (chaque femelle est conservée pour 
deux mises bas) et de l'ordre de $40 \mathrm{p}$. Ioo pour le renouvellement des mâles des noyaux (chaque mâle est utilisé pendant une période de saillie). Ces valeurs optimales varient peu avec la race du mâle terminal mais il est intéressant de noter que les petites différences trouvées vont dans le sens des conclusions de l'étude de MoAv et HiLl (I966) : les taux de sélection optimaux sur la prolificité sont d'autant plus faibles que la race paternelle a un effet plus défavorable sur la taille de portée (comparaison entre Large White "viande " et Piétrain) et que ses produits de croisement ont une valeur supérieure pour la performance de production (comparaison entre Landrace Belge et Piétrain).

Remarquons aussi que par rapport au croisement en retour où il n'y a pas de sélection sur la prolificité, le taux optimum de renouvellement est inchangé pour les mâles mais qu'il y a intérêt à conserver plus longtemps les femelles; Or.rivier (I974) est arrivé à un résultat analogue quand il a comparé les âges optimaux à la réforme des reproducteurs des deux sexes dans le cas de la sélection sur la performance de production et dans le cas de la sélection sur la taille de portée; dans ce dernier cas, la précision de la sélection est fonction du nombre de portées par femelle et donc de 1'âge de réforme des truies.

A l'origine de cette "interaction " entre plan de croisement et plan de sélection des noyaux "femelles ", il y a le fait que dans le croisement en retour, l'amélioration de la performance de production des produits terminaux dépend uniquement du progrès génétique des noyaux "femelles " de ce point de vue alors que dans le cas des croisements triples, seulement $50 \mathrm{p}$. Ioo des gènes des produits terminaux proviennent de ces noyaux. L'effort de sélection dans les noyaux "femelles " doit être consacré en totalité à l'amélioration de la performance de production dans le cas du croisement en retour. Par contre, il y a intérêt, dans le cas des croisements triples, à consacrer une partie de cet effort de sélection à 1'amélioration de la prolificité : en d'autres termes, l'utilisation d'une lignée mâle spécialisée, distincte des deux races maternelles, nécessite une plus grande spécialisation, par voie de sélection, de ces dernières.

En ce qui concerne la valeur relative des quatre plans de croisement, nos résultats appellent trois séries de commentaires.

La nature de ces résultats est étroitement dépendante des hypothèses que nous avons faites sur la valeur initiale des différents types génétiques pour les performances de reproduction et de production. Sur ce plan, deux effets, par leur ampleur même, ont une répercussion prépondérante sur la productivité de 1'étage terminal : 1'avantage du verrat Large White pour la taille de portée (de l'ordre de 7 p. roo) et la supériorité du verrat Landrace Belge pour la valeur de la marge unitaire de ses produits de croisement (comprise entre 8 et I2 p. Ioo). L'avantage du Large White sur les trois autres races, quant à l'effet de la race paternelle sur le nombre de porcelets sevrés dans les portées issues de truies $\mathrm{F}_{1}$, a été pris égal à 0,6 , chiffre qui correspond aux résultats tout à fait concordants de SMITH et KING (I964) et de VAN DE PAS et al. (I973); il semblerait, au vu de résultats plus récents (FENDER et al.; I975; CouANON, I977), que la supériorité réelle du verrat Large White de ce point de vue soit moins nette. Cet exemple illustre bien, à notre avis, les limites de telles études prévisionnelles sur les schémas d'amélioration génétique : les conclusions, ou au moins certaines d'entre elles, sont à considérer avec prudence, du fait de l'incertitude qui subsiste sur la valeur vraie d'un certain nombre de paramètres génétiques.

Il est intéressant de rapprocher les différences que nous avons trouvées entre plans de croisement pour le gain dû au croisement et celles qu'on peut déterminer de façon plus simple en s'inspirant de la méthode de MOAv (r966) : rappelons que 
cette méthode consiste à calculer la valeur d'une fonction de rentabilité (" profit function ") qui vise à estimer le bénéfice par porc produit dans l'étage terminal. En reprenant les mêmes paramètres génétiques et économiques, ce calcul conduit aux résultats suivants : le meilleur plan est le croisement triple avec le Landrace Belge (base roo), suivi dans l'ordre du croisement triple avec la souche Large White "viande " $(88,7)$, du croisement en retour $(77,9)$ et du croisement triple avec le Piétrain $(75,4)$. Le classement des quatre plans est donc inchangé par rapport à celui qui est fourni ici par le gain dû au croisement. Il y a également un bon accord entre les deux méthodes quant à la valeur relative des trois derniers plans mais, par contre, la fonction de rentabilité confère au croisement triple avec le Landrace Belge un avantage plus net que celui trouvé ici. En dépit de cette discordance dont la raison ne nous apparaît pas clairement, l'approche simplifiée qui consiste à déterminer la productivité du seul étage terminal permet, au moins dans un premier temps, de bien évaluer le mérite relatif de plusieurs plans de croisement pour le gain dû au croisement.

Enfin, - et c'est une autre conclusion qui mérite d'être soulignée - le gain dû au croisement ne suffit pas à caractériser le mérite d'une stratégie de croisement : ce gain traduit 1'efficacité immédiate du plan de croisement lui-même mais il y a lieu de tenir compte aussi des recettes qu'un effort de sélection "adapté " à ce plan permet d'espérer à long terme. Ainsi le croisement triple avec le Landrace Belge est, nous l'avons vu, la meilleure stragérie du point de vue du seul croisement mais si 1'on considère le bénéfice total calculé en supposant que, dans chaque cas, le plan optimum de sélection est pratiqué, le croisement triple avec la souche Large White "viande " donne les meilleurs résuitats, du fait d'une meilleure valorisation globale des actions de sélection, sur la période de $\mathrm{I} 5$ ans qui a été considérée.

Au-delà des résultats chiffrés qui ne donnent que des éléments de réponse partiels et dont certains, répétons-le, sont à interpréter avec prudence car ils reposent sur des hypothèses très fortes quant à leur incidence mais encore sujettes à caution, nous pensons que cette étude démontre la nécessité d'envisager conjointement l'optimisation du croisement et 1'optimisation de la sélection quand on recherche la meilleure stratégie à moyen et long terme pour l'amélioration génétique d'une espèce.

Reçu pour publication en décembre 1978 .

\section{Remerciements}

Les auteurs tiennent à remercier les deux lecteurs du manuscrit, le $D^{\mathbf{r}}$ M. BICHARD (Pig Improvement Company) et le $\mathrm{D}^{\mathrm{r}} \mathrm{W}$. ScHц, TE (Université d'Hohenheim) pour leurs utiles critiques et suggestions, ainsi que leurs collègues C. LEGAuLT, J. C. MocouoT et I. OLLIVIER pour l'aide apportée à différents stades de réalisation de cette étude.

\section{Summary}

$A$ joint study of the advantage of the selection on litter size and the use of a specialized sire line in the Pig

The joint optimization of several genetic decisions was studied in the case of a pig population which presents a three-tier structure of dissemination of genetic progress (selection, multiplication, production) and where a terminal two-stage cross is made involving Large White 
$(L W) \times$ French Landrace $(L F)$ dams. The number of these crossbred dams was supposed constant and the numbers of breeding animals in the upper tiers were adjusted to the replacement needs of the commercial herds, natural mating being only used. Two types of problems were investigated:

- choice of the terminal sive line: we compared the backcrossing system (using $L W$ and $L F$ boars equally) and three 3 -way crosses with a specialized sire line: Belgian Landrace, Pietrain and " $L W$ sive strain" different from the $L W$ dam strain.

- choice of the selection plan of the "female " nucleus herds ( $L W$ and $L F$ ) or, more precisely, determination of the optimum for the selection rates on litter size (using an index with variable precision) and the replacement rates of boars and sows.

The criterion to be maximized is the discounted profit brought by the production of pig meat over the whole "integrated "population. This profit, estimated for different discounting rates and periods of time, was splitted into two components: the "gain due to crossing " and the "gain due to selection". In the latter gain, we can distinguish returns due to the selection on production performance, returns due to the selection on litter size and returns due to the " joint effect " of these two selections.

Under our assumptions for the fixed parameters of the model, the main conclusions are:

- the optimum policy of selection in the "female " nucleus herds depends on the type of terminal cross. More particularly, selection on litter size is not of practical interest when making backcross but is justified in the 3-way crossing systems where applying optimum selection rates on litter size gives an increase of the gain due to selection, relatively to the case where litter size is not considered for selection: this increase may go up to $6 \mathrm{p}$. Ioo;

- the gain due to crossing is not sufficient for assessing the overall merit of a strategy of crossing. So the 3-way cross with Belgian Landrace is the best strategy from this view point but if we refer to the total profit, estimated with the assumption that in each case the optimum selection plan is applied, the 3 -way cross with " $L W$ sire strain " gives the best result, because selection work is more beneficial with that cross on the $\mathrm{r}_{5}$-year period considered.

\section{Références bibliographiques}

BerRuecos J. M., Dillard E. U., Robison O. W., I970. Selection for low backfat thickness in swine. J. Anim. Sci., 30, 844-848.

BICHARD M., I97I. Dissemination of genetic improvement through a livestock industry. Anim. Prod., 13, 40I-4II.

BICHARD M., I977. Economic efficiency of pig breeding schemes : a breeding company view. Livest. Prod. Sci., 4, 245-254.

BICHARD M., WILSON A., I974. Economic aspects of dissemination of genetic improvement throughout a crossbred pig structure. In Proceedings of the Working Symposium on Breed Evaluation and Crossing Experiment with Farm Animals, 289-296, Institut voor Veeteeltkunding Onderzoek "Schoonoord", Zeist, Pays-Bas.

BRUN J.-M., I974. Optimisation génétique et économique du croisement dans l'espèce porcine; application au cas du croisement entre les races Lavge White et Landrace. Mémoire de fin d'études', I.N.A. Paris-Grignon.

Chappuis P., Sourie J. C., I974. Modélisation de l'introduction d'un croisement à double étage dans un troupeau ovin. Document du Labovatoive d'économie rurale de Thiverval-Grignon, I.N.R.A., 34 pp.

Couanon Nicole, 1977. Performances de reproduction comparées en race pure et en croisement. Bulletin de l'Institut technique du Porc I977, 3, 9-I6.

Cunningham E. P., I973. Cost-effectiveness and population structure in cattle breeding programs. Ann. Génét. Sél. anim., 5, 239-256.

Dickerson G. E., I973. Inbreeding and heterosis in animals. In Proceedings of the Animal Breeding and Genetics Symposium in honor of Dr J. L. Lush, 54-77, A.S.A.S., A.D.S.A., Champaign, Illinois, U.S.A.

DICKERSON G. E., HAZEI L. N., I944. Effectiveness of selection on progeny performance as a supplement to earlier culling in livestock. $J$. agric. Res., 69, 459-476.

EIsEN J. M., Mocquo'T J. C., I974. Méthode de prévision de l'évolution du niveau gétiétique d'une population soumise à une opération de sélection et dont les générations se chevauchent. In Bull. tech. Dép. Génét. anim. (Inst. nat. Rech. agron., Fr.), no 17 , chap. II, 30-54. 
England M. E., Cunningham P. J., Mandigo R. W., Zimmerman R. W., i 977 . Selection for ovulation rate in swine : correlated response in carcass traits. J. Anim. Sci., 45, 983-988.

Faure P., Huard P., I965. Résolution de programmes mathématiques à fonction non linéaire par la méthode du gradient réduit. Revue Rech. opér., 36, 167-206.

FENDER M., RITTLER A., Schlote W., FEWSON D., I975. Ergebnisse des Hybridzuchtversuches in Baden-Württemberg. I. Mitteilung : Versuchsanlage und Zuchtleistungsprüfung. Schweinezucht- u. Schweinemast, 23, 60-64.

GARNETT I., RAHNEFELD G. W., I976. Mass selection for post-weaning growth in swine. V. Correlated response of reproductive traits and pre-weaning growth. Can. J. Anim. Sci., 56, 79 I-8OI.

Gray R. C., Tribble I. F., Day B. N., LASLEy J. F., I965. Five generations of selection for thinner backfat. J. Anim. Sci., 24, 848 (Abst.).

HAzel, I. N., Lush J. L., I942. The efficiency of three methods of selection. J. Hered., 33, 393-399.

HETZER H. O., MIILER R. H., I970. Influence of selection for high and low fatness on reproductive performance of swine. J. Anim. Sci., 30, $48 \mathrm{I}-495$.

HIL, W. G., 1974. Prediction and evaluation of response to selection with overlapping generations. Anim. Prod., 18, I 1 7-140.

JAKUBEC V., FEWSON D., I970. Ökonomische und genetische Grundlagen für die Planung von Gebrauchskreuzungen beim Schwein. 2. Mitteilung : Modellrechnungen über die Wirksamkeit verschiedener Formen von Gebrauchskreuzungen beim Schwein. Züchtungskunde, 42, 294-309.

LEGAULT C., r970a. Recherche d'un taux optimum de sélection des jeunes truies sur la prolificité de leur mère. In Journées de la Recherche Porcine en France 1970, 241-249, Institut technique du Porc, Paris.

LEGAULT C., r97o $b$. Étude statistique et génétique des performances d'élevage des truies de race Large White. II. Effet direct du verrat, héritabilité, répétabilité, corrélation. Ann. Génét. Sàl. anim., 2, 209-227.

LEGAUlT C., I97 Ia. Rôle de la sélection et des croisements dans l'amélioration de la productivité de la truie. Bull. tech. Inf. Ingrs Servs agric., 257, 221-229.

LEGAULT C., I97 I $b$. Corrélations entre les performances d'engraissement et de carcasse et les performances d'élevage chez le Porc. Ann. Génét. Sél. anim., 3, I 53-I6o.

LEGAUlT C., Dagorn J., TASTU D., I975. Effets du mois de mise bas, du numéro de portée et du type génétique de la mère sur les composantes de la productivité de la truie dans les élevages français. In Journées de la Recherche Porcine en France I975, XI,III-LII, Institut technique du Porc, Paris.

LEGault C., OLLIVIER L., I974. Plans de sélection porcine. In $x^{\text {er }}$ Congrès Mondial de Génétique Appliquée à l'Élevage (Madrid), vol. 1, 823-835, Editorial Garsi, Madrid.

Lush J. L., 1937. Animal Breeding Plans (I ${ }^{\text {re }}$ édition). Iowa State College Press, Ames, Iowa, U.S.A.

MOAv R., I966. Specialized sire and dam lines. I. Economic value of crossbreds. Anim. Prod., 8, I93-202.

MoAv R., HILL W. G., I966. Specialized sire and dam lines. IV. Selection within lines. Anim. Prod., 8, 375-387.

Molenat M., Houix Y., Poulenc J., I974. Contrôles d'engraissement et de carcasse en station chez les porcins : bilan et réflexions (1967-1973). Bull. tech. Dép. Génét. anim. (Inst. nat. Rech. agron., Fr.) no 18, ro4 pp.

MORRIS C. A., I975. Genetic relationships of reproduction with growth and with carcass traits in British pigs. Anim. Prod., 20, $3 \mathrm{I}-44$.

Newton J. R., CUnNingham P. J., ZImmerman D. R., I977. Selection for ovulation rate in swine: correlated response in age and weight at puberty, daily gain and probe backfat. $J$. Anim. Sci., 44, 30-35.

OLLIVIER L., I970. L'épreuve de la descendance chez le porc Large White français de I953 à I966. I. Analyse de la variation. Ann. Génét. Sél. anim., 2, 3 I I-324.

OLLIVIER L., I97I. L'épreuve de la descendance chez le porc Large White français de I953 à I966. II. Indices de sélection. Ann. Génét. Sél. anim., 3, 367-376.

OlLIVIER L., I974. Optimum replacement rates in animal breeding. Anim. Prod., 19, $257-27$ I.

Poutous M., VISSAC B., r962. Recherche théorique des conditions de rentabilité maximum de l'épreuve de descendance des taureaux d'insémination artificielle. Ann. Zootech., 11, 233-256.

SELLIER P., I974. Le croisement dans l'espèce porcine. In $I^{\text {er }}$ Congrès Mondial de Génétique Appliquée à l'Élevage (Madrid), vol. 1, 859-871, Éditorial Garsi, Madrid. 
SELLIER P., I975. Valeur en croisement de verrats Landrace Français et Piétrain. In Journées de la Recherche Porcine en France 1975, 285-292, Institut technique du Porc, Paris.

SELLIER P., I976a. Valeur en croisement de verrats Landrace Belge et Piétrain. In Journées de la Recherche Porcine en France 1976,22 I-228, Institut technique du Porc, Paris.

SELIIER P., 1976b. The basis of crossbreeding in pigs; a review. Livest. Prod. Sci., 3, $203-226$.

Selilier P., Houix Y., DESmoulin B., HenRy Y., I974. Premières observations sur la relation entre conditions nutritionnelles et type génétique chez des porcs femelles. In Journées de la Recherche Porcine en France 1974, 209-219, Institut technique du Porc, Paris.

Smiтh C., 1959. A comparison of testing schemes for pigs. Anim. Prod., 1, I I3-I 2 I.

Sмnтн C., 1964. The use of specialized sire and dam lines in selection for meat production. Anim. Prod., 6, 337-344.

Sмптн C., r978. The effect of inflation and form of investment on the estimated value of genetic improvement in farm livestock. Anim. Prod., 26, ror-I Io.

SmITH C., KING J. W. B., I964. Crossbreeding and litter production in British pigs. Anim. Prod., 6, 265-272.

STrang G. S., KING J. W. B., I970. Litter productivity in Large White pigs. 2. Heritability and repeatability estimates Anim. Prod., 12, 235-243.

VAN DE PAs J. G. C., Minkema D., Buiting G. A. J., I973. Results of crossbreeding experiments with pigs in the Netherlands. Ann. Génét. Sél. anim., 6, I49 (Abst.).

VANGEN O., 1972. Mortality in two lines of pigs selected for rate of gain and thickness of backfat. Acta Agr. Scand., 22, 238-242.

VANGEN O., r977. Correlated responses in a selection experiment with pigs after ro years of selection for growth rate and backfat thickness. $28^{\mathrm{e}}$ Réunion annuelle de la Fédération Européenne de Zootechnie, Bruxelles, I4 pp. (ronéoté).

WEBB A. J., KING J. W. B., I976. Development of a synthetic pig sire line by selection with immigration. I. Results of selection and heritability estimates. Anim. Prod., 22, 23 I-244. 